NASA CONTRACTOR REPOR T

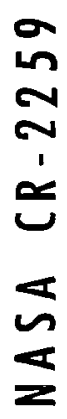

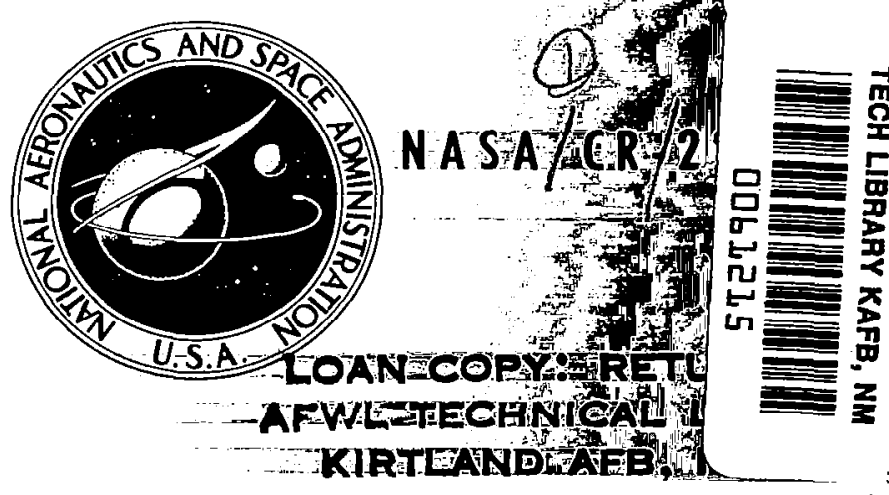

4 CALCULATION OF UNSTEADY

TRANSONIC AERODYNAMICS

FOR OSCILLATING WINGS

WITH THICKNESS

S. Y. Ruo and J. G. Theisen

\author{
Prepared by \\ 3 LOCKHEED-GEORGIA COMPANY \\ Marietta, Ga. 30060 \\ for Langley Research Center
}

NATIONAL AERONAUTICS AND SPACE ADMINISTRATION • WASHINGTON, D. C. • SAUGH 


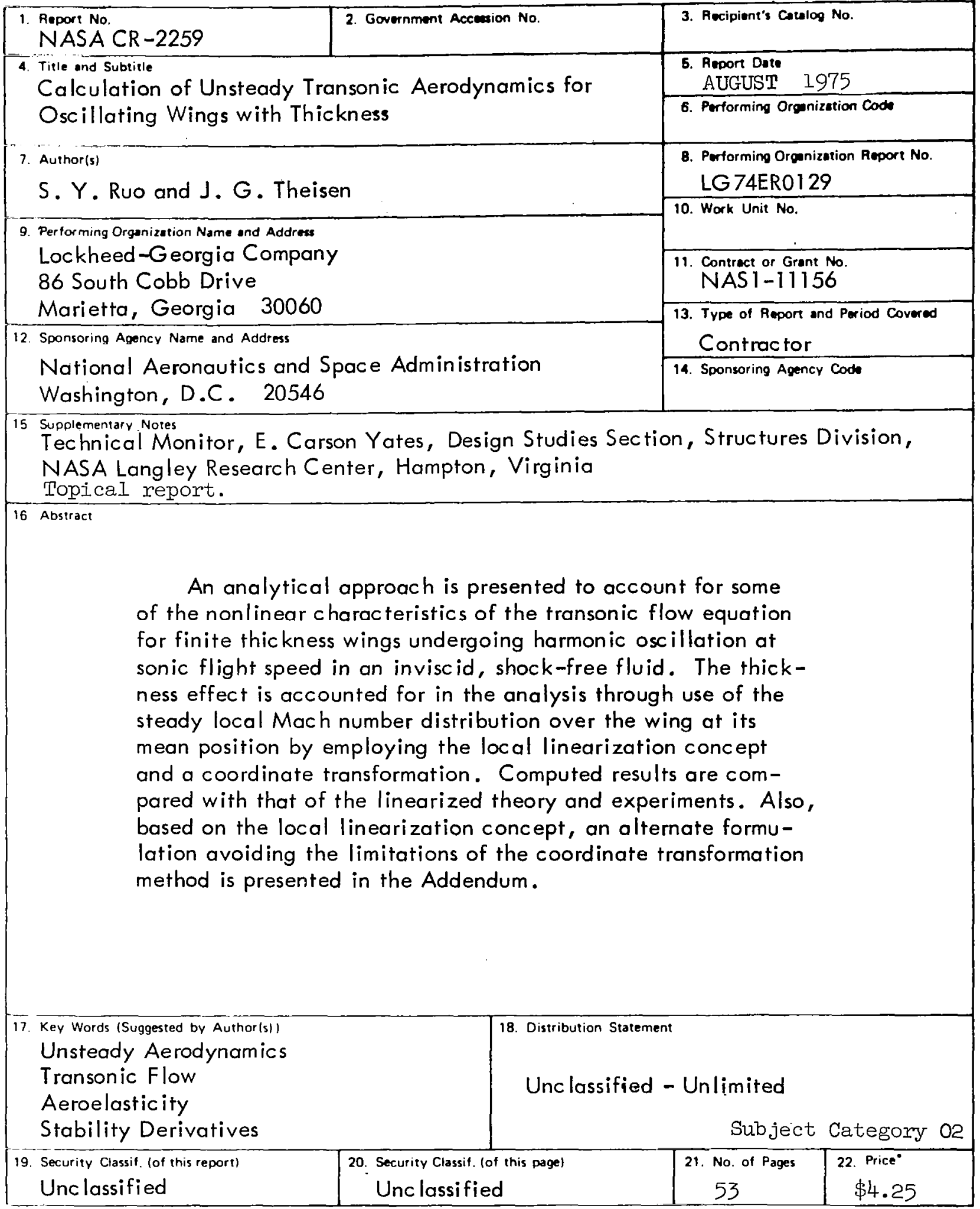

* For sale by the National Technical Information Service, Springfield, Virginia 21161 

SUMMARY

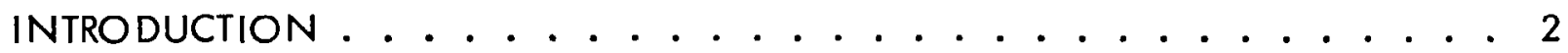

SYMBOLS ........................... 3

UNSTEADY TRANSONIC FLOW EQUATIONS. . . . . . . . . . . . . 6

The Basic Equation for Potential Flow. . . . . . . . . . . 6

Small Perturbation Potential Equation. . . . . . . . . . . . 6

Unsteady Small Perturbation Potential Equation . . . . . . . . . 8

Constant Coefficient Equation . . . . . . . . . . . . . . 9

Non-Constant Coefficient Equation . . . . . . . . . . . 10

Boundary Conditions . . . . . . . . . . . . . . . . . Il

Tangency Condition . . . . . . . . . . . . . . . 11

Pressure Coefficient . . . . . . . . . . . . . . 12

Forms of the Non-Linear Term in Steady Flow . . . . . . . . . . 13

Theoretical Methods to Obtain Numerical Results . . . . . . . . . 14

Linearized Method ... . . . . . . . . . . . . 14

Parabolic Method . . . . . . . . . . . . . . . . 14

Proposed Method . . . . . . . . . . . . . . . . 15

LOCAL LINEARIZATION. . . . . . . . . . . . . . . 15

Local Linearization Concept . . . . . . . . . . . . . . 15

Local Linearization Procedure. . . . . . . . . . . . . 16

Limitations of Proposed Transformation . . . . . . . . . . . 18

SONIC-BOX METHOD. . . . . . . . . . . . . . . . 19

Solution Procedure. . . . . . . . . . . . . . . . 19

Doublet Box Method . . . . . . . . . . . . . . . 20

DESCRIPTION OF THE COMPUTER PROGRAM .............. 21

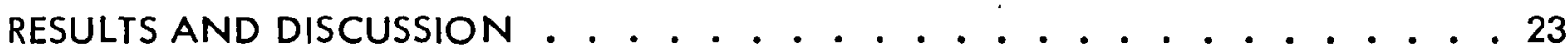

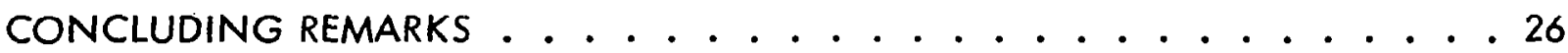


ADDENDUM: Alternate Modification of the Sonic-Box Method for Unsteady Finite Wings with Thickness Effects. . . . . . . . . . . . . 29

Summary . . . . . . . . . . . . . . . . . . . 29

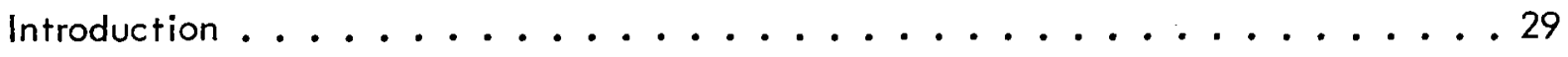

Equations and Solution . . . . . . . . . . . . . 30

Governing Equation . . . . . . . . . . . . . 30

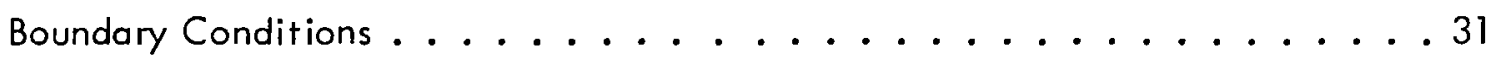

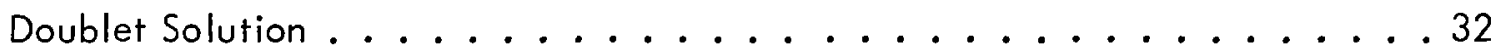

Doublet Sonic-Box Method . . . . . . . . . . . . . . . 33

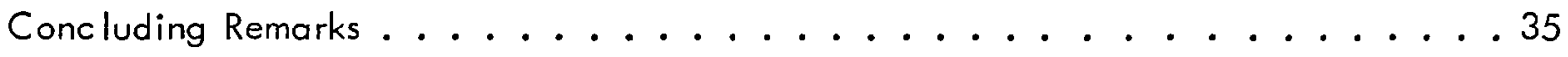

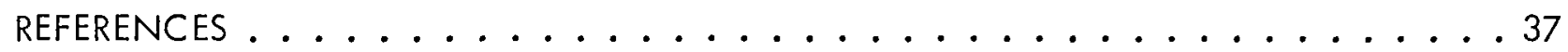

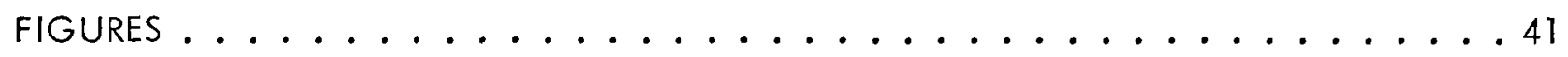




\title{
CALCULATION OF UNSTEADY TRANSONIC AERODYNAMICS \\ FOR OSCILLATING WINGS WITH THICKNESS*
}

\author{
By S. Y. Ruo and J. G. Theisen \\ Lockheed-Georgia Company
}

\section{SUMMARY}

An analytical approach is presented for determining some of the nonlinear characteristics of the transonic potential flow equation for finite thickness wings undergoing oscillatory motion at sonic flight speed in an inviscid, shock-free fluid. The thickness effect is accounted for in the analysis through the use of the steady local Mach number distribution on the wing, and corresponding mean flow parameters over the wing are considered to be constants in a small finite region. This latter assumption, equivalent to the concept of local linearization, permits the nonlinear differential equation for the velocity potential to be reduced to a linear one with variable coefficients containing the local Mach number. By means of an appropriate coordinate transformation, the equation becomes identical with the usual linearized transonic unsteady-flow equation with constant coefficients. Numerical results for wings with finite thickness can then be obtained by any computational technique that is suitable for use with the transonic linearized theory. For demonstration purposes herein, the well-known sonic-box computer program is adopted.

Stability derivatives and generalized aerodynamic force coefficients are shown for delta, rectangular and parabolic wing planforms over a range of reduced frequencies, with wing thickness and aspect ratio as parameters. Comparisons are made with available linearized theories and test data in the transonic flow regime, and reasonable agreement in trends is indicated. Relatively small thicknesses of 5 percent can have significant effects on generalized aerodynamic forces, e.g., on the order of 25 percent for rectangular wings of low aspect ratio, although the results for delta wings are much less. Still greater thickness levels may show a reversal of magnitude trend, according to these results, with corresponding effects possible for flutter instabilities.

\footnotetext{
* Portions of this report were included in AIAA Paper 73-316 which was presented at the AIAA Dynamics Specialists Conference, Williamsburg, Va., March 19-20, 1973.
} 


\section{INTRODUCTION}

- The study of flutter and other aeroelastic responses of an aircraft requires adequate knowledge of the forc es acting on three-dimensional wings in oscillatory motion. Such aeroelastic problems are frequently critical in the transonic speed range. The physical problem is governed by a nonlinear partial differential equation with nonlinear boundary conditions, for which an exact solution is not known to exist. The basic small perturbation equation governing the velocity potential for transonic flow over a thin wing or a slender body is well known (e.g., refs. 1 and 2). However, the nonlinearity which remains present prevents closed-form solutions from being obtained except in a few special cases (ref. 1). For a low-amplitude, high-frequency oscillation where the unsteady part is considered to be a small disturbance to the steady part, the steady-state properties can be completely uncoupled from the unsteady equation, and the governing equation for unsteady transonic flow can be linearized (refs. 2 and 3 ).

Almost all unsteady transonic flow theoretical work lies within this framework of linearized theory where the thickness effect of the wing is neglected. However, oscillatory transonic aeroelastic instabilities of lifting surfaces (i.e., flutter) often occur at frequencies below the range of validity of the transonic linearized theory, thus indicating a need to account for at least the more prominent effects of finite thickness. Recent studies (ref. 4) show that wing thickness, which enters into the mathematical nonlinearity, can significantly affect unsteady pressures in the supersonic range also. The object of this work, therefore, is to develop an appropriate method of accounting for the more important effects of finite wing thickness in order to predict transonic oscillating aerodynamic parameters at frequencies lower than those for which linearization is valid. The present study is limited to attached, shock-free flow.

An important consequence of the previously mentioned linearization is the suppression of deviations in local Mach number from freestream value. Since these deviations have appreciable effect on propagation of pressure disturbances over the lifting surface, significant improvement in the theory may be accomplished by "recoupling" the steady and unsteady flow parameters in a manner that at least accounts approximately for variations in mean local Mach number caused by finite wing thickness. In the present development, this is achieved by considering all of the steady-flow parameters over the wing to be invariant within a small finite region. This latter assumption, equivalent to the concept of local linearization, permits the nonlinear differential equation for the velocity potential to be reduced to a linear equation with variable coefficients containing the local Mach number. By means of an appropriate coordinate transformation, the equation becomes identical with the usual linearized transonic unsteady-flow equation with constant coefficients. Hence, numerical results for a lifting surface in the transformed space may be obtained by any computational technique that is suitable for use with the transonic linearized theory. For demonstration purposes herein, the well-known sonic-box computer program (refs. 5 and 44) is adopted. 
Since this investigation is exploratory, the limitations on wing planform geometry imposed by the sonic-box method of reference 5 have not been removed. The types of wings which can be treated are those having unswept trailing edges without control surfaces. Also, because of the transformation method used, the steady flow every where over the wing must not be very different from that of the freestream. The mean angle of attack, twist and camber of the wing are considered to be small, and the Mach number distribution on the wing is assumed to depend only on the wing thickness. Therefore, the mean local Mach number on a corresponding point of the upper and lower wing surfaces is considered to be the same in the present work. However, the extension of the present method to wings with different Mach number distributions on the upper and lower surfaces is self-evident:

Calculations using the present method were made to evaluate the thickness contribution to the unsteady aerodynamic forces by comparison with cases not having any thickness effect. The wings considered were: (a) rectangular wings with a bicircular-arc profile; (b) delta wings with an elliptic lateral cross-section; and (c) parabolic wings with a double-wedge lateral cross-section. Comparisons are made with the son ic box method, for cases without thickness effects, as well as with available test data on finite thickness wings. $(r e f, 6)$.

A detailed description of the computer program is available as a supplementary report

\section{SYMBOLS}
A
aspect ratio or influence coefficient
a
dimensionless pitch axis location measured positive aft from most forward point on wing (reference length $=b$ )
B
$B(x, y, z, t)=0$ defines the dimensionless body surface at any instant (reference length $=b$ )
b reference length - wing centerline chord (dimension $=L$ )
c local speed of sound (dimension $=L / T$ )
$\mathrm{C}_{\mathrm{L}_{\alpha}} \quad$ lift-curve-slope
$\mathrm{C}_{M_{\dot{\alpha}}}+\mathrm{C}_{M_{q}}$ damping-in-pitch
$C_{p}$
pressure coefficient
$\mathrm{D} / \mathrm{D} \boldsymbol{t}$
substantial derivative
$\mathrm{E}$
box approximation of the wing planform
$e, \exp$
exponential function
$f$
dimensionless mode shape
H
dimensionless length of box side (reference length $=b$ ) 
dimensionless distance from $z=0 \quad$ (reference length $=b$ )

i $\sqrt{-1}$

$\vec{i}, \vec{i}, \vec{k}$

unit vectors in $x-, y-, z$-direction, respectively

Im

imaginary part of the complex quantity

k

reduced frequency, $\omega b / U_{\infty}$

$L$

unit of length

$L_{i j}$

generalized aerodynamic force coefficient

M

local Mach number

$\theta$

reads "order of"

$q$

local flow speed, $\sqrt{u^{2}+v^{2}+w^{2}} U_{\infty} \quad($ dimension $=L / t)$

$\operatorname{Re}$

real part of the complex quantity

dimensionless planform area for full wing (reference area $=b^{2}$ )

s

dimensionless local semi-span (reference length $=b$ )

$T$

unit of time

t

dimensionless time (reference time $=b / \mathrm{U}_{\infty}$ )

$U_{\infty}$

freestream velocity (dimension $=L / T$ )

$u, v, w$

dimensionless velocity components in $x-, y-, z$-direction, respectively ( $r$ eference velocity $=U_{\infty}$ )

W

wake

$x, y, z$ dimensionless cartesian coordinates (reference length $=b$ )

a angle of attack

ᄃ acceleration parameter

y ratio of specific heats

$\nabla \quad \vec{i} \frac{\partial}{\partial x}+\vec{i} \frac{\partial}{\partial y}+\vec{k} \frac{\partial}{\partial z}$, dimensionless gradient operator 

denotes sonic condition

denotes dimensional quantity or operator

(i.e., ()$_{\text {dimensional }}=()_{\text {dimensionless }} \times$ (reference quantity))

denotes vector quantity

denotes transformed quantity

'," denote first and second total differentiations

UNSTEADY TRANSONIC FLOW EQUATIONS AND SOLUTIONS

The Basic Equation for Potential Flow

The exact, nonlinear, unsteady flow equation to be satisfied by the velocity potential $\Omega$, as given in reference 7 , is

$$
\bar{\nabla}^{2} \Omega-\frac{1}{c^{2}}\left[\frac{\partial^{2}}{\partial \bar{t}^{2}}(\Omega)+\frac{\partial}{\partial \bar{t}}\left(q^{2}\right)+\vec{q} \cdot \bar{\nabla}\left(\frac{1}{2} q^{2}\right)\right]=0,
$$

where $\vec{q}=\bar{\nabla} \Omega$ and $c$ is the local speed of sound. All quantities in equation (1) are dimensional.

\section{Small Perturbation Potential Equation}

In order to obtain the small perturbation velocity potential equation, the small perturbation potential $\Phi$ is introduced by writing

$$
S(\bar{x}, \bar{y}, \bar{z}, \bar{f})=U_{\infty}[\bar{x}+\bar{\Phi}(\bar{x}, \bar{y}, \bar{z}, \bar{t})],
$$

then, the velocity components are expressed as

$$
\left.\begin{array}{l}
\bar{u}=\Omega_{x}=U_{\infty}\left(1+\bar{\Phi}_{\bar{x}}\right) \\
\bar{v}=\Omega_{\bar{y}}=U_{\infty} \bar{\Phi}_{\bar{y}} \\
\bar{w}=\Omega_{\bar{z}}=U_{\infty} \bar{\Phi}_{\bar{z}}
\end{array}\right\}
$$


The substitution of equation (3) into equation (2), with the introduction of the following set of dimensionless variables

$$
x=\frac{\bar{x}}{b}, y=\frac{\bar{y}}{b}, z=\frac{\bar{z}}{b}, t=\frac{U_{\infty} \bar{t}}{b} \text {, and } \Phi=\frac{\bar{\Phi}}{b}
$$

results in the expression,

$$
\begin{aligned}
\nabla^{2} \Phi-\frac{U_{\infty}^{2}}{c^{2}}\left[\Phi_{x x}+2 \Phi_{x t}+\Phi_{t t}\right]=\frac{U_{\infty}^{2}}{c^{2}} & \left\{2\left(\Phi_{x} \Phi_{x t}+\Phi_{y y t}+\Phi_{z} \Phi_{z t}\right)\right. \\
& +2\left(\Phi_{x} \Phi_{x x}+\Phi_{y} \Phi_{y x}+\Phi_{z} \Phi_{z x}\right) \\
& +\Phi_{x}\left(\Phi_{x} \Phi_{x x}+\Phi_{y} \Phi_{y x}+\Phi_{z} \Phi_{z x}\right) \\
& +\Phi_{y}\left(\Phi_{y x y}+\Phi_{y} \Phi_{y y}+\Phi_{z} \Phi_{z y}\right) \\
& \left.+\Phi_{z}\left(\Phi_{x} \Phi_{x z}+\Phi_{y} \Phi_{y z}+\Phi_{z} \Phi_{z z}\right)\right\} .
\end{aligned}
$$

Commonly, the perturbation is assumed to be so small that the quadratic and cubic terms on the right-hand side of equation (4) may be neglected in comparison with the linear term on the left-hand side. Thus, equation (4) simplifies to

$$
\left(1-\frac{U_{\infty}^{2}}{c^{2}}\right)_{x x}+\Phi_{y y}+\Phi_{z z}-\frac{U_{\infty}^{2}}{c^{2}}\left(2 \Phi_{x t}+\Phi_{t t}\right)=0 .
$$

This may be considered as a linearized equation with variable coefficients. A further simplification is usually made by replacing the local speed of sound with that of the freestream by employing the Bernoulli equation:

$$
\frac{c^{2}}{u_{\infty}^{2}}=\frac{c_{\infty}^{2}}{u_{\infty}^{2}}-(Y-1)\left[\Phi_{\dagger}+\Phi_{x}+\frac{1}{2}(\nabla \Phi)^{2}\right] .
$$

Thus, equation (5) becomes the well-known differential equation of acoustic theory

$$
\left(1-M_{\infty}^{2} \Phi_{x x}+\Phi_{y y}+\Phi_{z z}-2 M_{\infty}^{2} \Phi_{x t}-M_{\infty}^{2} \Phi_{t t}=0 .\right.
$$

The equation of motion has been considerably simplified by using the small perturbation method; but this linearized equation does not always yield appropriate solutions for some 
physical flow phenomena in transonic flow. In the study of transonic flow a nonlinear term is normally retained to account for the nonlinear effects which usually occur in this flow regime. Landahl (ref. 2) derived the small perturbation potential equation for unsteady transonic flow by retaining the first- and second-order term contribution from the $x$-derivative term in the continuity equation and only the first-order term contribution from the $y-, z-$, and $t$-derivative terms. The resulting equation is

$$
\left[\left(1-M_{\infty}^{2}-M_{\infty}^{2}(Y+1) \Phi_{x}\right] \Phi_{x x}+\Phi_{y y}+\Phi_{z z}-2 M_{\infty}^{2} \Phi_{x t}-M_{\infty}^{2} \Phi_{t t}=0\right.
$$

If the non-constant coefficient in equation (5) is approximated by

$$
\frac{u_{\infty}^{2}}{c^{2}}=\frac{q^{2}}{c^{2}} \frac{U_{\infty}^{2}}{q^{2}} \cong M^{2}\left(1-2 \Phi_{x}\right)
$$

and only the first order terms are retained, equation (5) becomes

$$
\left(1-M^{2}\right)_{x x}+\Phi_{y y}+\Phi_{z z}-M^{2}\left(2 \Phi_{x t}+\Phi_{t t}\right)=0 \text {. }
$$

This equation maintains the mixed flow property usually observed in the transonic flow regime. It is not, however, in the form commonly used in transonic flow studies because of the presence of non-constant coefficients. Use of the freestream Mach number may be physically justified in place of the local Mach number in equation (9) as long as the local Mach number is not very different from unity. That is, both equations (7) and (9) are acceptable based on the analysis, but equation ( 7 ) does not preserve the mixed flow properties of the transonic flow problem. Thus, instead of equation (7), equation (8) is being used more frequently.

Both equations (8) and (9) are capable of representing mixed flow but neither equation is easily tractable as required to satisfy the boundary conditions. These include the requirements that the small perturbation velocity vanishes at infinity and the flow must be tangent to the solid surface. Also, in unsteady flow studies, the equation is further simplified and linearized by assuming that the unsteady potential is a small perturbation over that of the steady part.

\section{Unsteady Small Perturbation Potential Equation}

Under the assumption that the unsteady perturbation potential is small relative to that of the steady part, the steady and unsteady parts in the small perturbation equation may be completely separated when the unsteadiness (e.g., the frequency of oscillation) is high. Subsequently, the unsteady form of the equation is usually linearized. 
Constant Coefficient Equation.- Following Landahl (ref. 2) the small perturbation potential $\Phi$ may be written as

$$
\Phi(x, y, z, t)=\phi(x, y, z)+\varphi(x, y, z, t) .
$$

Then, for the case of oscillatory motion where the amplitude is much smaller than the thickness ratio, equation ( 8 ) becomes

$$
\left(1-M_{\infty}^{2}\right) \phi_{x x}+\phi_{y y}+\phi_{z z}=M_{\infty}^{2}(\gamma+1) \phi_{x} \phi_{x x}
$$

and

$$
\left(1-M_{\infty}^{2}\right) \varphi_{x x}+\varphi_{y y}+\varphi_{z z}-2 M_{\infty}^{2} \varphi_{x t}-M_{\infty}^{2} \varphi_{t t}=M_{\infty}^{2}(\gamma+1) \frac{\partial}{\partial x}\left(\phi_{x} \varphi_{x}\right),
$$

where equations (11) and (12) correspond, respectively, to steady and unsteady transonic flows. Equation (11) involves only the steady flow quantities but equation (12) contains a coupling term between the steady and unsteady quantities. The term $\varphi_{x} \varphi_{x x}$ has been ignared because $\varphi$ is considered to be a perturbation on $\phi$, so $\varphi_{x} \varphi_{x x}$ is a higher order term than $\varphi_{x} \phi_{x x}$ or $\varphi_{x x} \phi_{x}$.

Landahl (ref. 2), following the fundamental development of Lin, et al. (ref. 8), showed that with the assumptions that $\varphi_{t}=\theta^{\prime}\left(k \varphi_{x}\right)$ and $k \gg\left|\phi_{x}\right|$ everywhere in the flow, the coupling term on the right-hand side of equation (12) can be neglected. Thus equation (12) becomes linearized and completely decoupled from the steady flow quantity, as follows:

$$
\left(1-M_{\infty}^{2} \varphi_{x x}+\varphi_{y y}+\varphi_{z z}-2 M_{\infty}^{2} \varphi_{x t}-M_{\infty}^{2} \varphi_{t t}=0\right. \text {. }
$$

Since in transonic flow $1-M_{\infty}=\sigma\left(\phi_{x}\right)$, equation (13) may be further simplified to

$$
\varphi_{y y}+\varphi_{z z}-2 M_{\infty}^{2} \varphi_{x t}-M_{\infty}^{2} \varphi_{t t}=0
$$

Equation (14) is valid when

$$
|1-M| \cong \phi^{\prime}\left(\phi_{x}\right)
$$

In reference 9, Miles presented an excellent discussion of the conditions for the simplification of the small perturbation equation in various flow regimes.

The freestream Mach number $M_{\infty}$ in equation (14) can be eliminated by a transformation using $Y=M_{\infty} y$ and $Z=M_{\infty} Z$ so that only the case when $M_{\infty}=1$ needs to be considered. That is, for sonic flow, 


$$
\varphi_{y y}+\varphi_{z z}-2 \varphi_{x t}-\varphi_{t t}=0
$$

When the wing is performing oscillatory motion, the unsteady small perturbation potential can be expressed as

$$
\varphi(x, y, z, t)=\varphi_{0}(x, y, z) e^{i \omega \bar{t}}=\varphi_{0}(x, y, z) e^{i k t}
$$

and equation (14) becomes

$$
\varphi_{o_{y y}}+\varphi_{o_{z z}}-M_{\infty}^{2} k\left(2 i \varphi_{o_{x}}-k \varphi_{o}\right)=0
$$

Non-constant Coefficient Equation.- If the steady local Mach number $M$ is taken to be the mean local Mach number, equation (9), with the relation in equation (10), can be easily written as

$$
\left(1-M^{2}\right) \phi_{x x}+\phi_{y y}+\phi_{z z}=0
$$

and

$$
\left(1-M^{2}\right) \varphi_{x x}+\varphi_{y y}+\varphi_{z z}-M^{2}\left(2 \varphi_{x t}+\varphi_{t t}\right)=0,
$$

where equations (18) and (19) govern, respectively, the steady and unsteady flow.

By following the same reasoning used in obtaining equation (14) a similar equation can be derived for unsteady flow with non-constant coefficients, namely

$$
\varphi_{y y}+\varphi_{z z}-M^{2}\left(2 \varphi_{x t}+\varphi_{t t}\right)=0,
$$

or

$$
\varphi_{\sigma_{y y}}+\varphi_{o_{z z}}-M^{2} k\left(2 i \varphi_{o_{x}}-k \varphi_{0}\right)=0
$$

Equation (21) is the basic equation considered in the present study, to investigate thickness effects on the aerodynamic forces, in which the steady local Mach number is treated as a parameter. 


\section{Boundary Conditions}

Tangency Condition.- For an inviscid fluid flowing over a solid surface, it is assumed that at the surface the normal component of the relative velocity is zero and slip may occur; there is no boundary layer separation and no shock formation. Thus the tangency condition can be written as (ref. 10)

$$
\frac{D \bar{B}}{D \bar{f}}=0
$$

where $\bar{B}(\bar{x}, \bar{y}, \bar{z}, \bar{f})=0$ defines the body surface position at any instant, and

$$
\frac{D}{D \bar{f}}=\frac{\partial}{\partial \bar{t}}+\bar{u} \frac{\partial}{\partial \bar{x}}+\bar{v} \frac{\partial}{\partial \bar{y}}+\bar{w} \frac{\partial}{\partial \bar{z}} .
$$

For a small perturbation flow, equation (22) can be written in dimensionless form as

$$
B_{t}+\left(1+\Phi_{x}\right) B_{x}+\Phi_{y} B_{y}+\Phi_{z} B_{z}=0 \text {, on } B=0 \text {. }
$$

To the first order, for a general thin body $\left(\Phi_{x} \ll 1\right)$, equation (23) can be approximated by

$$
B_{t}+B_{x}+\Phi_{y} B_{y}+\Phi_{z} B_{z}=0 \text {, on } B=0 \text {, }
$$

and for a planar body, equation (24) can be further simplified if only the first order terms are retained:

$$
B_{t}+B_{x}+\Phi_{z}=0, \quad \text { on } z=0
$$

Equation (25) is the linearized tangency condition for the planar case.

For a thin body performing a small oscillation from its mean position, the instantaneous position of its upper surface may be approximated by (fig. 1)

$$
z=h_{1}(x, y)+h_{2}(x, y, t)
$$

or

$$
B(x, y, z, t)=z-h_{1}(x, y)-h_{2}(x, y, t)
$$

By substituting equation (26) into equation (24), one obtains 


$$
\Phi_{z}=h_{1_{x}}+\left(h_{2 x}+h_{2}\right)+\Phi_{y}\left(h_{1}+h_{2}\right), \quad \text { on } B=0
$$

Since the body under consideration is thin and its departure from the mean position is small, the spatial derivatives of $h_{1}$ and $h_{2}$ may be considered to have the same order of magnitude and $\Phi_{y}$ is much less than unity; thus, equation (27) may be linearized (i.e., eq. (25)). When equation (10) is introduced in the left-hand side of the linearized form of equation (27), one arrives at

and

$$
\left.\begin{array}{l}
\phi_{z}=h_{x} \\
\varphi_{z}=h_{2}+h_{2}
\end{array}\right\} \quad \text { on } z=0
$$

where the steady and unsteady tangency conditions have been uncoupled.

For a body performing oscillatory motion

$$
h_{2}(x, y, t)=h_{0}(x, y) e^{i \omega \bar{t}}=h_{0}(x, y) e^{i k t},
$$

the linearized unsteady tangency condition in equation (28) may be written as

$$
\varphi_{o_{z}}=h_{o_{x}}+i k h_{o^{\prime}} \text { on } z=0 \text {. }
$$

Pressure Coefficient.- The linearized pressure coefficient written in terms of the small perturbation potential is

$$
C_{p}(x, y, 0, t)=-2\left(\Phi_{t}+\Phi_{x}\right) \text {. }
$$

Substitution of the relationship in equation (10) into equation (30), with $C_{p}$ written as a linear combination of the steady and the unsteady parts, results in

$$
C_{p_{1}}(x, y, 0)=-2 \phi_{x} \quad \text { (steady part) }
$$

and

$$
C_{p_{2}}(x, y, 0, t)=-2\left(\varphi_{x}+\varphi_{t}\right) \quad \text { (unsteady part) }
$$

If equation (16) is substituted in equation (32) the following expression is obtained 


$$
c_{p_{0}}=-2\left(\varphi_{o_{x}}+i k \varphi_{0}\right)
$$

where

$$
C_{p_{0}}(x, y, 0)=C_{p_{2}}(x, y, 0, t) e^{-i k t}
$$

For locations in the wake of a wing, $C_{p}=0$, or

$$
\varphi_{0_{x}}+i k \varphi_{0}=0
$$

\section{Forms of the Nonlinear Term in Steady Flow}

In order to mainta in the mixed-type flow in the study of steady transonic flow, the variable coefficient of the first term in equation (18) is often approximated by the general form

$$
\left(1-M^{2}\right)=\left(1-M_{\infty}^{2}\right)-K \phi_{x},
$$

where $K$, which represents the coefficient of the nonlinear term, assumes several forms as listed below:

$$
\begin{aligned}
& K=(Y+1) M_{\infty}^{2} \quad \text { (e.g., ref. } 11 \text { or eq. (11)) } \\
& K=(Y+1) \quad(\text { e.g., refs. 12, 13, 14, 15) } \\
& K=M_{\infty}^{2}\left[2+(Y-1) M_{\infty}^{2}\right] \quad(\text { e.g., ref. 16) }
\end{aligned}
$$

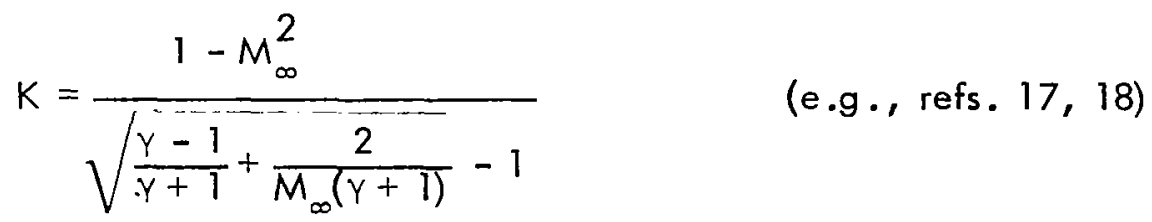

$$
\begin{aligned}
& K=\frac{1}{\phi_{x x}} M_{\infty}^{2}(Y+1) \phi_{x x}\left(x^{*}\right) \quad(\text { e.g., refs. 19, 15, 20) } \\
& \left.K=\frac{(\gamma+1)\left[B^{\prime \prime}\left(x^{*}\right) \cdot \ln \tau\right]\left(x-x^{*}\right)}{\phi_{x}} \quad \text { (e.g., ref. } 21\right) \text {. }
\end{aligned}
$$

Equation (38) can be obtained by expressing $M^{2}$ in terms of $M_{\infty}^{2}$ and utilizing the Bernoulli equation relating the local and freestream speed of sound, and retaining the first 
order terms. When $M_{\infty}$ tends to unity, equations $(36),(38)$, and (39) all reduce to equation (37). Equation (39) was obtained from the mass-flow consideration. Equations (40) and (41) were obtained more or less intuitively. Equation (40) forms the basis for the parabolic method. Equation (41) was needed to treat the body of revolution for the case of $M_{\infty}=1$ only; the form of $\mathrm{K}$ would be different when the freestream Mach number is not unity.

When the wing is of very small aspect ratio and $1-M=\sigma\left(\phi_{x}\right), K$ may be set to zero (e.g., refs. 2,22$)$.

Theoretical Methods to Obtain Numerical Results

In all the practical unsteady transonic flow studies to date, only the sonic freestream flow case has been considered. This is because the linearized equation with constant coefficients, namely, equation (13) has been used and a simple transformation can be used to eliminate $M_{\infty}$ in the equation. Then a similarity rule can be applied to obtain information for those cases where Mos is slightly different from unity. The linearized theory has been used with some success in comparison with test data. But due to the possible presence of wind tunnel wall interference, shock formation, and flow separation it is sometimes difficult to make a direct. comparison of the test data with the theoretical results and to identify the source of disagreement. Because of this, only the theoretical methods leading to numerical results are mentioned in the following paragraphs and no attempt is made to evaluate the validity of each method.

Linearized Method.- Mangler (ref. 23) used the pressure doublet distribution to treat the delta wing at sonic speed, for small $k$, by letting $M_{\infty}$ approach unity from the subson ic solution. Miles analyzed the low aspect-ratio rectangular wing at sonic speed in reference 24. Later, Runyan and Woolston (ref. 25) solved the case of an oscillating rectangular wing at sonic speed by extending the kernel function method for general planforms in subsonic flow. In reference 2, Landahl extensively treated the delta and rectangular wings by solving the boundary value problem in a Fourier transformed plane. Using the delta wing solution as a tool, Landahl also studied low aspect-ratio wings, wing-body combinations, as well as wings of general form composed of straight segments. Davies (ref. 3) used lifting surface theory to treat general planforms for large $k$. An elliptic cone performing plunging oscillations at sonic speed was investigated by Albano and Andrew (ref. 26) for the effects of blunt leading edges and wing thickness.

Rodemich and Andrew (ref. 5) developed a sonic-box method for non-swept trailing edge wings with arbitrary wing planforms; and, later, Stenton and Andrew (ref. 27) extended the method to include swept trailing edges with control surfaces.

Parabolic Method.- In the linearized method the unsteady flow part has been completely uncoupled from the steady part; that is, the thickness effect has been neglected. An 
attempt to take the coupling of the steady part into account, at least to the first order, has been made by Teipel (ref. 28) for two-dimensional flow and Liu et al. (refs. 29, 30) for axisymmetrical flow at sonic speed. This is based on the parabolic method concept (eq. (40)) introduced by Oswatitsch and Keune (ref. 19) for steady axisymmetrical flow. An equation in the form of equation (12) is used with the term $\phi_{x} \varphi_{x x}$ ignored and with $\phi_{x x} \varphi_{x}$ written as $\Gamma \varphi_{x} /\left[M_{\infty}^{2}(Y+1)\right]$.

Proposed Method.- It has been shown by Kacprzynski, et al. (ref. 4), for supersonic flow that thickness effects on the lift, aerodynamic center, and flutter speed of a delta wing could be very significant. It is not difficult to conceive that the thickness could also have a marked effect on the aerodynamic forces on a finite wing over the transonic flow range. The thickness effect is introduced through the use of the local Mach number of the wing at its mean position in the unsteady, small perturbation equation.

The proposed equation to be used in this investigation is equation (20) in which $M$ is the steady local Mach number representing the effect due to wing thickness. The solution to equation (20) satisfying the required boundary conditions is obtained by using the concept of local linearization attributed to Spreiter and Alksne (ref. 31) as described in the following section.

\section{LOCAL LINEARIZATION}

\section{Local Linearization Concept}

The thickness of the airfoil, the formation of shocks, the interaction between the turbulent boundary layer and the shock, and the presence of separated flow may all contribute important effects on the loading. The actual physical problem is governed by nonlinear partial differential equations with nonlinear boundary conditions, and defies exact solution. Hence, we confine ourselves here to an extension of the linear theory only to account for the effect of wing thickness insofar as it produces a nonuniform mean flow and, possibly, a small locally supersonic region. This should be a significant improvement over the usual idealized lifting surface model.

One method of improving the linearized theory is to account for the nonlinearity in an approximate fashion, based on the concept of "local linearization." In this method, the effect of thickness is included only to the extent that the flow remains free of shocks, The underlying assumption is that the physical state, usually governed by nonlinear equations, is adequately described by related linear equations, with all parameters involved having their local values taken to be constants in a limited region. This involves replacement of the nonlinear equations with linear equations having variable coefficients. 
These methods were introduc ed into aerodynamics in an intuitive way for steady flow by Spreiter and Alksne (ref. 31), and have now been rigorously validated by means of the method of parametric differentiation by Rubbert and Landahl (ref. 32). This approach suggests that, in the case of unsteady flow, the calculations can be carried out with sufficient accuracy using the linearized equations which contain the local values of the steady-flow parameters. In his linearized theory, Landahl (ref. 33) cites evidence for the validity of applying the concept of local linearization to the case of unsteady flow.

Moreover, in the case of supersonic flow, it was pointed out by Ashley (ref. 34) that a potentially simple way of improving the accuracy of unsteady-flow calculations is to use the linearized velocity-potential equation, but with the Mach number of the undisturbed flow replaced by the local value which varies spatially due to thickness, mean angle of attack, and/or camber-line shape. This work has been extended in two ways. Sankaranarayanan and Vijayavittal (ref. 35) applied Ashley's (ref. 34) approach to flow past delta wings and found that the general effect of thickness is to reduce the flutter speed; meanwhile, Kacprzynski (ref. 36) examined the three-dimensional effects more fully. The above investigations are concerned with studying thickness effects in supersonic flow. Except for the work of Andrew and Stenton (ref. 37) relatively little information is available about the behavior of a finite wing with thickness in the transonic flow regime.

It should be pointed out that one still earlier method for analyzing the transonic airfoil problem also introduces a concept similar to that of local linearization. Using the theoretically exact hodograph transformation, Garrick and Kaplan (refs. 38, 39) developed velocity correction formulas which provide a basis for comparison of corresponding compressible and incompressible flows. In reference, 40, Guderley discusses the extension of the hodograph method to the problem of three-dimensional transonic flow around finite-span wings. However, the advantage of linearization is lost in the extension to three dimensions, and his results are limited to certain trends for the influence of aspect ratio for a special type of finite-span wing. Guderley concludes that to find a complete solution of the nonlinear equation for transonic flow around a wing of moderate aspect ratio would prove extremely cumbersome, if not impossible.

\section{Local Linearization Procedure}

The local linearization procedure starts with the unsteady transonic flow equation, equation (20), namely

$$
\varphi_{y y}+\varphi_{z z}-M^{2}\left(2 \varphi_{x t}+\varphi_{t t}\right)=0,
$$

where the local Mach number $M$ is given by

$$
M=M_{\infty}\left[1-\frac{1}{2}\left(1+\frac{\gamma-1}{2} M_{\infty}^{2}\right) C_{p_{1}}\right] \text {. }
$$


Here, $C_{P_{1}}$ is the pressure coefficient of the wing associated with the steady flow at its mean position. $M$ or $C_{P_{1}}$ will have to be provided either from theoretical results or from available test data. The quantity $M$ is all that is required to proceed with the sonic-box method solution (ref. 5).

For oscillatory motion, equation (42) can be written as (see, e.g. eq. (21))

$$
\varphi_{o_{y y}}+\varphi_{o_{z z}}-M^{2} k\left(2 i \varphi_{o_{x}}-k \varphi_{o}\right)=0
$$

Then, by introducing the following modified Prandtl-Glavert transformation

$$
\left.\begin{array}{l}
\tilde{y}=M(x, y) \cdot y \\
\tilde{z}=M(x, y) \cdot z
\end{array}\right\}
$$

into equation (44) and neglecting the spatial derivatives of $M$ (here $M$ is considered as a parameter), one obtains the following

$$
\tilde{\varphi}_{o \sim} \tilde{y}_{y}+\tilde{\varphi}_{o \sim z z}-2 i k \tilde{\varphi}_{o_{x}}+k \tilde{\varphi}_{0}^{2 \sim}=0
$$

where

$$
\tilde{\varphi}_{0}(x, \tilde{y}, \tilde{z})=M(x, y) \varphi_{0}(x, y, z)
$$

This equation is in the form that Rodemich and Andrew (ref. 5) used in their sonic-box method. Thus a wing properly transformed according to equation (45) may be treated by the sonic-box method in the transformed space. Even though the local Mach number may be different from unity on a given wing, the consideration of $M$ here is not thought to be the primary effect which changes the type of flow; rather, it is considered to be a parameter, with the local disturbances unable to travel upstream for the cases under study by virtue of the Mach freeze.

The corresponding linearized tangency condition becomes

$$
\tilde{\varphi}_{\sigma_{\tilde{z}}}=\tilde{h}_{o_{x}}+i k \tilde{h}_{0}
$$

where

$$
\tilde{h}_{0}(x, \tilde{y})=h_{0}(x, y)
$$




\section{Limitations of the Proposed Transformation}

The sonic-box method developed by Rodemich and Andrew (ref. 5) is restricted to a wing where no part of it is downstream of any part of its wake(s). That is, the slope of the leading edges of the wing $d s / d x$ is not allowed to change signs as shown in figure 2 . The leading edge shown in the positive $\tilde{y}$ quadrant is unacceptable because its slope changes sign between the apex and the trailing edge of the wing. While the slope of the leading edge shown in the negative $\tilde{y}$ quadrant does not change sign, thus it is acceptable. Since the sonic-box method is applied to the transformed wing in the present study, this restriction may be expressed as

$$
\frac{d}{d x}[ \pm \widetilde{s}(x)] \geq 0,
$$

or, written in the quantities for the physical plane,

$$
\pm s(x)=-M( \pm s) \cdot \frac{\frac{d}{d x}[ \pm s(x)]}{\frac{d}{d x}[M( \pm s)]},
$$

where $M( \pm s)$ represents the local Mach number along the leading edges.

In addition to the limitation shown in equation (50), there is another limitation concerning the spanwise Mach number distribution. That is, at $x=$ constant,

$$
\frac{d \tilde{y}}{d y}>0 \quad \text { for } \quad 0<|y|<s \text {. }
$$

Figure 3 illustrates the nature of the acceptable and the unacceptable functional forms. In the sketch, the relation $\tilde{y}=y \cdot M(x, y)$ is drawn as a solid line. As can be seen in the illustration, the transformed local span in $\tilde{y} \geq 0$ is single-valued (acceptable) whereas that in $\tilde{y}<0$ is multi-valued (unacceptable). The multi-valued transformation creates a type of fold-over wing in the transformed space which makes this method inapplicable. This condition may be relaxed somewhat if at those points where the values of $d \bar{y} / d y=0$ occur within the range $|\widetilde{y}|<|\widetilde{s}|, \widetilde{y}$ only deviates slightly from the required monotonically increasing nature as $y$ is transformed into $\tilde{y}$ from 0 to $s$. 
Although any computational technique that is suitable for use with the linearized transonic theory could be applied in combination with the present method, the basic computer program chosen for use in this study was that originally developed by Rodemich and Andrew (ref. 5) and known generally as the "sonic-box" program. Since equation (46) and the boundary condition, equation (49), to be treated in the transformed space are in the form that was used in reference 5, the principal task required to account for the thickness effect is the transformation of information between the physical and the transformed spaces according to equations (45), (47), and (49). The mathematical development of the numerical procedure of carrying out the integration will not be duplicated completely here; however, the pertinent equations are included for the purpose of continuity. Since the wing to be considered is in the transformed space, the quantities in the transformed space rather than that of the physical space are used in the description of the sonic-box method.

\section{Solution Procedure}

The basic solution satisfying equations (20) and (29) for a thin wing having its mean position lying in $\times \widetilde{y}$-plane (see fig. 4) representing a point doublet with its axis oriented in $z$-direction is

$$
\widetilde{\varphi}_{d}(x, \tilde{y}, \tilde{z})=\left\{\begin{array}{l}
0, x \leq 0 \\
\frac{i k}{2 \pi} \frac{\widetilde{z}}{x^{2}} \cdot \exp \left[-\frac{i k}{2}\left(x+\frac{\widetilde{y}^{2}+\widetilde{z}^{2}}{x}\right)\right], x>0 .
\end{array}\right.
$$

Then the solution to equation (20) for a distribution of doublets can be written as

$$
\tilde{\varphi}_{0}(x, \tilde{y}, \tilde{z})=\left\{\begin{array}{l}
0, x \leq 0 \\
\int_{x-\xi>0} \int_{\rho} \rho(\xi, \eta) \cdot \widetilde{\varphi}_{d}(x-\xi, \tilde{y}-\eta, \widetilde{z}) d \xi d \eta, x>0
\end{array}\right.
$$

where $\rho(x, \tilde{y})$, representing the doublet strength, may be any function such that the integral exists.

The downwash for a point $(x, \widetilde{y})$ on $\widetilde{z}=0^{+}$of the wing mean plane, may be obtained from equation (52) 


$$
\widetilde{w}\left(x, \tilde{y}, 0^{+}\right)=\lim _{\widetilde{z} \rightarrow 0^{+}} \frac{\partial}{\partial \widetilde{z}}\left|\tilde{\varphi}_{0}\right|=\lim _{\widetilde{z} \rightarrow 0^{+}} \frac{\partial}{\partial \widetilde{z}}\left\{\iint \rho(\xi, \eta) \cdot \widetilde{\varphi}_{d}(x-\xi, \widetilde{y}-\eta, \widetilde{z}) d \xi d \eta\right\}
$$

or

$$
\widetilde{w}\left(x, \tilde{y}, 0^{+}\right)=\iint_{\widetilde{W}} \int_{\widetilde{S}} \rho(\xi, \eta) \cdot \psi\left(x-\xi, \widetilde{y}-\eta, 0^{+}\right) d \xi d \eta
$$

where

$$
\begin{aligned}
\psi\left(x, \tilde{y}, 0^{+}\right)= & \lim _{\widetilde{z} \rightarrow 0^{+}} \frac{\partial}{\partial \widetilde{z}}\left[\tilde{\varphi}_{d}(x, \tilde{y}, \widetilde{z})\right] \\
& = \begin{cases}0, & x \leq 0 \\
\frac{i k}{2 \pi} \frac{1}{x^{2}} \cdot \exp \left[-\frac{1}{2} i k\left(x+\frac{\widetilde{y}^{2}}{x}\right)\right], & x>0\end{cases}
\end{aligned}
$$

and

$$
\rho(x, \widetilde{y})=\widetilde{\varphi}_{0}\left(x, \tilde{y}, 0^{+}\right)
$$

Thus the boundary value problem becomes

$$
\tilde{w}\left(x, \tilde{y}, 0^{+}\right)=\iint \tilde{\varphi}_{0}\left(\xi, \eta, 0^{+}\right) \cdot \psi(x-\xi, \tilde{y}-\eta) d \xi d \eta, \text { for }(x, \tilde{y}) \text { in } \widetilde{S},
$$

and from equation (34)

$$
\left(\frac{\partial}{\partial x}+i k\right) \tilde{\varphi}_{0}\left(x, \tilde{y}, 0^{+}\right)=0, \text { for }(x, \tilde{y}) \text { in } \widetilde{W}
$$

Doublet Box Method

The amplitude of oscillation of the moderately thin wing under consideration is assumed to be small. The mean position of the wing is considered to lie approximately in the $x \widetilde{y}-$ plane with its nose at the origin and with an unswept trailing edge. The freestream is assumed to be parallel to the $x$-axis and at a Mach number of unity. The value of the unsteady potential on the wing may be found by using equation (54).

To get an approximate solution of equation (54), let the $x \tilde{y}$-plane be covered with a grid of square boxes with the box edges parallel to the $x$-and $y$-axis. Let the region $E$ be composed of all boxes whose centers lie in $\widetilde{S}$. Thus, $E$ is an approximation to $\widetilde{S}$ by boxes. The potential $\widetilde{\varphi}_{0}$ at each box is assumed to be constant, i.e., $\widetilde{\varphi}_{0_{i j}}=$ constant at $(i, j)$-th box $E_{i j}$. The tangency condition is applied to the center $\left(x_{i}, y_{i}\right)$ of each box $E_{i j}$ in $E$ and the region of integration is replaced by $E$. Thus equation (54) gives a system of linear 
algebraic equations for the $\widetilde{\varphi}_{\mathrm{o}_{i j}}$ 's:

$$
\sum_{i^{\prime}, i^{\prime}} \widetilde{\varphi}_{o_{i^{\prime} i^{\prime}}} \iint_{E_{i^{\prime} i^{\prime}}} \psi\left(x_{i}-\xi, \widetilde{y}_{i}-\eta\right) d \xi d \eta=\widetilde{w}\left(x_{i}, \widetilde{y}_{i}\right)
$$

or

$$
\sum_{i^{\prime}} A\left(0,\left|i-i^{\prime}\right|\right) \widetilde{\varphi}_{o_{i i^{\prime}}}=\widetilde{w}\left(x_{i^{\prime}}, \tilde{y}_{i^{\prime}}\right)-\sum_{i^{\prime}<i} \sum_{i^{\prime}} A\left(i-i^{\prime},\left|i-i^{\prime}\right|\right) \widetilde{\varphi}_{o_{i^{\prime}} i^{\prime}}
$$

where

$$
A\left(i-i^{\prime},\left|i-i^{\prime}\right|\right)=\iint_{E^{\prime} i^{\prime}} \Psi\left(x_{i}-\xi, \tilde{y}_{i}-\eta\right) d \xi d \eta .
$$

If the wing is symmetric about the $x$-axis, then only modes of oscillation that are symmetric or antisymmetric in y need be treated. Thus one only needs to consider one-half of the wing in a computation. For symmetric modes, equation (57) becomes

$$
\begin{gathered}
\sum_{i^{\prime} \geq 1}\left[A\left(0,\left|i-i^{\prime}\right|\right)+A\left(0, i^{\prime} i^{\prime}-1\right)\right] \widetilde{\varphi}_{o_{i j}} \\
=\widetilde{w}\left(x_{i}, \widetilde{y}_{i}\right)-\sum_{i^{\prime}<i} \sum_{i^{\prime} \geq 1}\left[A\left(i-i^{\prime},\left|i-i^{\prime}\right|\right)+A\left(i-i^{\prime},\left|i^{\prime}+i^{\prime}-1\right|\right] \widetilde{\varphi}_{o_{i} i^{\prime}}, i \geq 1 .\right.
\end{gathered}
$$

The equations for $j \leq 0$ are implied by $i \geq 1$.

For antisymmetric modes, equation (59) applies, with the sums of values of $A$ replaced by differences.

\section{DESCRIPTION OF THE COMPUTER PROGRAM}

A major part of the computer program used in this study is adapted from that developed by Rodemich and Andrew in reference 5 . The capability of the original sonic-box computer program is retained and, in addition, it has been extended to include nonlinear thickness effects. This is accomplished in an approximate manner by applying the local linearization concept in conjunction with the mean local Mach number distribution which is assumed known.

The wing with thickness is transformed into a wing without thickness in the transformed space using the relations in equation (45). The equation to be solved and the boundary 
condition to be satisfied in the transformed space become those shown in equations (46) and (49), respectively. Then the sonic-box method is applied directly, since the linearized theory is applicable in the transformed space.

The leading edge of the planforms of both the physical and transformed wings must not have any local maximum in the spanwise direction within the range $0 \leq x<b ; i . e$. , the local semi-span must not decrease as $x$ increases from the nose to the trailing edge. The leading edge of any given wing planform is represented by a finite number of straight line segments and, based on this, the area is then approximated by a grid of square boxes. In each box, represented by its center, the flow parameters are considered to be constants equal to their values at the box centers. The trailing edge of the wing must be unswept*, and there must be no control surfaces. The computer program as presently formulated considers only the symmetric mode * of oscillation, having the xz-plane as the plane of symmetry, and the local Mach number at a corresponding point on the upper and lower wing surfaces is taken to be the same.

The doublet potential at the center of each box in the transformed space is obtained by using equation (54), with a known downwash, from the sonic-box computer program. Then it is converted into the doublet potential of the physical wing by applying equation (47). That is, the doublet potential at the center of each box in the physical space is found by locating the corresponding point in the transformed space and applying the inverse transformation. The inversion results in a non-uniform array, but this method applies interpolation with a polynomial fitting procedure to the data before further usage for integrations. Equation (33) is used in the calculation of the unsteady pressure coefficient and the generalized cerodynamic force coefficients are computed from the following expression (ref. 2):

$$
L_{i j}=\frac{4}{S \delta_{i}} \iint_{S}\left(\varphi_{o_{x}}+i k \varphi_{o}\right)_{i} f_{i} d x d y,
$$

where the oscillatory displacement in the $z$-direction from the mean position of the wing is written as

$$
h_{2}(x, y, t)=h_{0}(x, y) e^{i k t}=\delta_{i} f_{i}(x, y) e^{i k t}
$$

so that the mode shape can be related as $f_{i}(x, y)=\frac{h_{0}(x, y)}{\delta_{i}}$,

with $h_{0}$ as the nondimensional amplitude in $z$-direction, and $\delta_{j}$ as the nondimensional amplitude of the $i^{\text {th }}$ mode.

The stability derivatives are calculated by using the following relationships:

* This restriction is carried over from reference 5 and is not an essential limitation of the present method. 


$$
\begin{gathered}
C_{L_{\alpha}}=-\frac{1}{k} \operatorname{lm}\left[L_{11}\right] \\
C_{M_{\dot{\alpha}}}+C_{M_{q}}=\frac{1}{k} \operatorname{lm}\left[L_{22}-a\left(L_{21}+L_{12}\right)+a^{2} L_{11}\right] .
\end{gathered}
$$

The standard output of the computer program consists of the generalized aerodynamic force coefficients. In addition to these, the Mach number, downwash, potential, as well as the lifting pressure distributions can be printed out if they are desired.

\section{RESULTS AND DISCUSSION}

Typical unsteady aerodynamic results have been calculated, using the above local linearization procedure, for a variety of wing planforms and thicknesses. Such studies are necessarily limited by the availability of steady-state data on local pressure or Mach number distributions for any desired wing shape or thickness, since this is required as input to the basic calculations. The planforms selected for this study are rectangular, delta and parabolic, and the steady state Mach number distributions over these wings in a sonic flow field are available from reference 41 . This information is furnished to the computer in the form of pressure coefficient or Mach number distributions at a group of selected points on the wing. The data are then fitted with a pre-selected polynomial form representing the Mach number as a function of $x$ and $y$ using the least-square method available in the original sonic-box program.

The standard output from the analysis is in the form of amplitude and phase of the generalized aerodynamic force coefficients as a function of frequency. Typical results are shown in figures 5 and 6 for rectangular and delta wings, respectively. The rectangular wing has a biconvex airfoil section, with an aspect ratio $A=2.0$, and is pitching about an axis along the leading edge. Based on a distribution of 25 boxes along the centerline chord, parts (a) and (b) of figure 5 indicate that for sonic flow conditions, quite large reductions in generalized force coefficients are possible for rectangular wings of thickness $\tau=.0521$. However, changes as great as 25 percent are larger than anticipated, especially at the higher frequencies. In the absence of directly correlated tests, with finite thickness rectangular wing models, these results should be used with caution since some limitations of the program are more pronounced for this case. The steady Mach number distribution is quite rapidly varying, particularly near the leading edge of this wing (ref. 41), so in the transformed coordinates the planform is severely distorted. The box size used may be too large to properly define these variations. Also, the requirement of the linearized theory that the reduced frequency satisfy the relationship $k \gg\left|\phi_{x}\right|$ is probably violated by the large velocity disturbances near the leading edge. This may explain the apparent lack of convergence in magnitude for the two $\left|L_{11}\right|$ coefficients in figure $5(a)$ at frequencies near $k=1.0$. At high frequency, the linearized theory is expected to be accurate, independent of thickness considerations. However, the residual differences between the two results for the higher $k$ values used here may exist because that maximum $k$ value is still insufficiently high to insure 
convergence. This possibility may best be seen in figure $5(\mathrm{~b})$ where good convergence is exhibited by the phase angle $\theta_{22}$, and $\left|L_{22}\right|$ shows some asymptotic tendency at the highest frequency calculated. Further study of the effects of box size is needed for rectangular wing cases.

A typical variation of the generalized aercdynamic force coefficient for pitching moment due to pitch oscillations of a delta wing configuration of aspect ratio $A=1.5$ are shown in figure 6. A distribution of 40 boxes along the centerline chord was used in the computations. This wing has an elliptic cross-section in a plane perpendicular to the chordwise axis, and it is assumed to be pitching about the apex, or the most forward point of the delta wing; i.e., $a=0$. Lift and pitching moment magnitudes and phase angles show very little change with thickness increase as compared with the rectangular wing values. At medium-to-high reduced frequencies, increases in absolute values are of the order of 5 percent, with the maximum occurring for 10 percent thickness. Over most of the frequency range, a further increase to 15 percent thickness results in a reversal of trend, or less increase in magnitude of the force coefficients. As will be discussed later, however, variation of pitch axis to more aft locations may affect the percent change markedly for a delta wing.

The magnitude of the unsteady pressure distributions for this delta wing are presented in figure 7 for both pitching and plunging motions at reduced frequency 0.1 . The pressure variation for a change from zero to 10 percent maximum thickness is not very large except near the trailing edge. The changes are generally greatest where anticipated based on consideration of local flow acceleration due to the addition of thickness.

Comparisons are made in figure 8 with the low-frequency approximation in Landahl's linearized theory (ref. 2, chapter 3) for a delta wing without thickness effect. The trends of increasing damping-in-pitch $\left(\mathrm{C}_{\mathrm{M}_{\dot{\alpha}}}+\mathrm{C}_{\mathrm{Mq}}\right)$, with increasing frequency are qualitatively in good agreement for the higher aspect ratio case. For the lower reduced frequencies, larger effects on damping-in-pitch are possible due to changes in either thickness or pitch axis location, as shown in figures 8 and 9 . The differences in absolute magnitudes for the zero thickness cases, particularly at higher frequencies, may be rationalized by considering limitations of both linearized methods compared in the figures. Landahl's approximate low aspect-ratio method should be adequate at moderately low frequencies for these two cases $(A=1.5$ and $A=3.0)$. His solution has an upper frequency limitation, and this may explain part of the differences in figure 8. However, the local linearization method also has a severe limitation since the potential differences between the "locally constant" function over each of the boxes do not cancel at the edges of adjacent boxes when the integration is carried out around the boxes. This implies some error in the surface integral which increases with the size of the box used. This may explain the lack of convergence with Landahl's linearized theory at the higher frequencies.

As mentioned previously, the delta wing thickness does not appear to affect the results as much as for the rectangular wing at the higher reduced frequencies. It is believed, however, that some additional effects would be seen if an improved fitting procedure were to be employed. The steady flow results used (ref . 41) indicate a less rapidly varying function over the surface of the delta wing, but the least-square fitting method in the original 
sonic-box program essentially reduces the pre-determined polynomial into a linear form. The more rapid variations at the leading and trailing edges were ignored. Thus, the transformed planform was only slightly different from that for the delta wing, and only small thickness effects were therefore observed. Further study is planned using improved fitting techniques to determine more accurately the sensitivity of the program to delta wing thickness variations.

Only a small amount of test data are available for oscillatory transonic flow with these configurations. The data scatter is generally great, but the more reliable appearing data at $M_{\infty}=1$ in references 42 and 43 are shown in figures 9,10 , and 11 . The data for delta wings with finite thickness indicate that both trend and magnitudes of the stability derivative shown in figure 9 may be in reasonably good agreement at intermediate reduced frequencies. The chordwise pressure distributions plotted in figure 10 show better agreement at the outer wing stations. Data from the same tests (ref. 43) in figure $11(b)$ also show good agreement with the analysis for lift-curve slopes as a function of frequency. Trends with wing thickness are not observable because of scatter in the test data.

Only results based on thin-wing theory are available for comparison with the rectangular and delta wing calculations in figures 11 (a) and $11(b)$, respectively. Landahl's lowfrequency approximation (ref. 2, chapter 4) compares quite well for the rectangular wing in figure $11(\mathrm{a})$ at low reduced frequencies. Similarly, Davies' theory (ref. 3) agrees very well with results for the delta wing at aspect ratio, $A=1.5$, shown in figure $11(b)$.

The capability of the program to represent other planforms is demonstrated in figure 11 (c), where a six-segment approximation to the leading edge of a parabolic wing and a distribution of 40 boxes along the centerline chord were used. The cross-section consists of a double wedge in a plane perpendicular to the chordwise axis. For this case, as for others mentioned, the polynomial representing the Mach number distribution on the wing obtained from the leastsquare method was not believed to be of adequate accuracy. Improved procedures are available for fitting such functions, and it is recommended that the program be checked for each case until modifications can be made in the future.

Another question concerning the sonic-box procedure in present use is concerned with the convergence of the magnitude and phase angle as related to the number of boxes used to represent the planform. A typical study which should be made for each new configuration is shown in figure 12. It is apparent that the generalized force coefficient converges adequately for the 40 boxes employed in the delta wing cases studied herein. However, at higher frequencies it may be desirable to use a larger number of boxes to properly represent the wing planform. For the case with thickness effect, this may be especially needed because of the greater complexity of the leading edge of the transformed wing.

Because of the transformation technique adopted, certain types of wings for which a oneto-one transformation cannot be made must be excluded from treatment by the present approach. However, this difficulty can be alleviated by using a locally varying source strength distribution, related to the local Mach number, in place of the transformation presently employed. 
Quantitative evaluation of the local linearization procedure introduced here for unsteady flow cannot be completed until other theories with transonic thickness effects are developed, or until more reliable test data become available for comparison.

\section{CONCLUDING REMARKS}

The local linearization concept is applied to the determination of approximate wing thickness effects in unsteady transonic flow using the sonic-box computational procedure. The principal argument in this concept is that the mean-flow properties in a sufficiently small region on a wing may be treated as essentially constant. Thus, the governing potential-flow equation becomes linear with variable coefficients. A coordinate transformation then reduces this equation to linear form with constant coefficients, which is in the same form as the wellknown linearized transonic-flow equation. Hence, numerical results in the transformed space may be obtained by any computational technique that is suitable for use with the transonic linear theory. For demonstration purposes herein, the well-known sonic-box computer program is adopted.

Sample calculations for delta, rectangular and parabolic wings are made to demonstrate the capabilities of the program and to show the contribution of wing thickness effects to various unsteady flow quantities. Comparisons are made with results from linearized theories as well as with available test data, and reasonable trends with thickness effects are indicated.

The results for. low aspect-ratio delta wings, having elliptical spanwise cross-sections and pitching around the apex, indicate that thickness causes rather small changes in pressure, damping-in-pitch, and generalized force coefficients relative to those calculated from linearized theory using the sonic-box method. However, for this particular wing, these quantities do not vary monotonically with thickness; so this trend reversal needs further study with other cross-sectional geometries and other pitch axis locations before firm conclusions can be made. The zero thickness results for both the delta and the rectangular wings are in reasonable agreement with the corresponding theory by Landahl for low aspect ratios over the lower and medium range of reduced frequencies.

Although calculations for a low aspect-ratio rectangular wing indicated thickness effects as large as 25 percent, caution should be exercised in interpreting these results since certain limitations of the computer program and the transformation used, in their adopted forms, are most pronounced for this configuration. The wing forms which can be treated with the present program are limited to those having a mean local Mach number distribution not very different from the freestream value. Those wings which cause a multi-valued transformation (i.e., a fold-over wing in the transformed space) must be excluded. In addition, only unswept trailing edges without control surfaces are allowed by the present computational technique. 
The fold-over limitation and edge conditions associated with the transformation method can be removed by using a modified source strength distribution, related to the local Mach number, in place of the transformation presently employed to account for thickness effects. Also, improved accuracy may be obtained by replacing the least-square surface-fitting procedure used in the original sonic-box computer program with better approximations.

The limited amount of test data available for delta wings in unsteady transonic flow show reasonably good agreement with the analysis for finite thickness cases and for variations with reduced frequency. Absolute magnitudes cannot be evaluated very well until more reliable test data are obtained. 


\title{
ADDENDUM
}

\section{ALTERNATE MODIFICATION OF THE SONIC-BOX METHOD FOR UNSTEADY FINITE WINGS WITH THICKNESS EFFECTS}

\author{
By S. Y. Ruo
}

\section{SUMMARY}

An analytical approach is presented for determining some of the nonlinear characteristics of the transonic flow equation for finite thickness wings undergoing oscillatory motion at sonic flight speed in an inviscid, shock-free fluid. The thickness effect is introduced into the basic solution through the variation of the mean local Mach number on the wing, and the flow parameters over the wing are considered to be constants within a small finite region. Thus, the nonlinear differential equation can be reduced to a locally linear one with variable coefficients.

The equations required to obtain numerical results are reduced to forms similar to those used in the well-known sonic-box method computer program. Thus, with some minor modifications to the original computer program, the transonic flow aerodynamic force coefficients with thickness effects can easily be obtained.

\section{INTRODUCTION}

It is well known that a closed-form solution to the small perturbation equation governing unsteady transonic potential flow over a finite wing is not easily attainable. The previous developments for dealing with the problem of transonic flow over an oscillating wing have largely been restricted to linearized theories (see e.g. refs. 2 and 3 ). These methods are a result of uncoupling the unsteady motion from the steady part and are generally limited to high frequency cases. Since the steady part is completely uncoupled from the unsteady equation, the thickness effects cannot be accounted for by using the linearized theory.

In the work by Kacprzynski, et al. (ref. 4), it has been indicated that thickness, which enters into the mathematical nonlinearity, can significantly affect unsteady pressure in the supersonic range. More recently, efforts to approximately incorporate the thickness effect in unsteady transonic flow analysis have been made by several investigators (refs. $28,29,30,45$, and 46). Among the approaches used are extensions of the concepts leading to the parabolic and local linearization methods introduced for steady transonic flow, respectively, by Oswatitsch and Keune (ref. 19) and by Spreiter and Alksne (ref. 31). 
In the analysis based on the parabolic method concept (refs. 28, 29, 30, 45, and 46), the thickness effect is considered in a global sense and is determined by the overall body shape at its mean position. This approach can be improved by modifying the global thickness effect to a locally dependent one to refine the physical interpretation of the thickness effect in the unsteady transonic flow analysis. In the main part of the present work, which is based on the local linearization concept, the thickness effect is considered in terms of the mean local Mach number. That is, instead of the freestream Mach number, the mean local Mach number is used in the governing equation, which is then a linear equation with variable coefficients. With an appropriate coordinate transformation in conjunction with the local linearization concept, the equation is converted to one with constant coefficients, and the well-known sonic-box method (ref. 5) is used in the transformed space to obta in the numerical results. During the course of this study, it was found that the transformation used was not always adequate, especially when the Mach number variation over the wing is large. In such a case, a one-to-one transformation may become impossible, thus limiting the capability of the above technique to account for thickness effects in unsteady transonic flow analysis. In both approaches, the parabolic and the coordinate transformation methods, the thickness effect is considered to be independent of the body unsteadiness, and only the steady conditions at the mean wing position are taken into account. Additionally, the solutions are limited to $M_{\infty}=1.0$, zero mean angle of attack, shock-free attached flow, and wings with trailing edges which are unswept and without control surfaces.

The purpose of this Addendum is to investigate the possibility of formulating the problem directly from the doublet potential expressed in terms of the local Mach number and, hence, avoid some of the limitations of the transformation method which were discussed in the main portion of this report.

Symbols, references, and figures are as in the main portion of this report.

\section{EQUATIONS AND SOLUTION}

\section{Governing Equation}

The small perturbation equation governing unsteady transonic flow as considered here for $M_{\infty}=1.0$ in dimensionless form is

$$
\varphi_{y y}+\varphi_{z z}-2 M^{2} \varphi_{x t}-M^{2} \varphi_{t t}=0
$$

and for harmonic motion, equation $(A-1)$ becomes

$$
\varphi_{o_{y y}}+\varphi_{o_{z z}}-2 i k M^{2} \varphi_{o_{x}}+k^{2} M^{2} \varphi_{0}=0,
$$


where $\varphi=\varphi_{0} e^{i k t}$.

Equation $(A-2)$ is a linear equation with variable coefficients. The local Mach number on the wing at its mean position is independent of the motion.

The coordinate system used is shown in figure 4.

\section{Boundary Conditions}

The boundary conditions to be satisfied are: (1) any disturbances must vanish at infinity, and (2) the flow must be always tangent to the solid surface. The first condition is satisfied by the type of solution chosen, and the second condition can be written as

$$
\frac{D}{D t} B(x, y, z, t)=0 \text {. }
$$

The linearized tangency condition for the unsteady part is obtained as

$$
\varphi_{o_{z}}=h_{o_{x}}+i k h_{0} \text { on } z=0
$$

for harmonic motion.

The linearized tangency condition shown in equation (A-4) has been completely uncoupled from the steady part, and this implies that the mean angle of attack, camber, and twist of the wing are small (fig. l).

For $M_{\infty}=1.0$, as in the linearized case, it is assumed that no disturbances propagate upstream. Even though the mean local Mach number on the wing may be greater than, or less than, unity, the implication of the procedure is that the locally subsonic or supersonic character of the flow is not the predominant influence on the oscillatory potential.

$$
\begin{aligned}
& \varphi_{o_{z}}\left(x, y, 0^{+}\right)=h_{o_{x}}+i k h_{0}, \text { for }(x, y) \text { in } S \\
& \varphi_{o_{x}}\left(x, y, 0^{+}\right)+i k \varphi_{0}\left(x, y, 0^{+}\right)=0, \text { for }(x, y) \text { in } W \\
& \varphi_{0}\left(x, y, 0^{+}\right)=0, \text { for }(x, y) \text { not in }(S+W) .
\end{aligned}
$$




\section{Doublet Solution}

The basic solution, representing a point doublet oriented parallel to the z-axis at the origin and satisfying the required condition at infinity for a small finite region on the wing where the value of $M$ is considered to be constant, may be written for equation $(A-2)$ as

$$
\varphi_{d}(x, y, z ; M)= \begin{cases}0, & x \leq 0 \\ \frac{i k}{2 \pi} & \frac{z M^{2}}{x^{2}} \exp \left\{-\frac{1}{2} i k\left[x+\frac{M^{2}\left(y^{2}+z^{2}\right)}{x}\right]\right\}, x>0,\end{cases}
$$

in which $M$ is regarded as a parameter rather than a flow influencing property.

Equation (A-6) satisfies equation (A-2) only in a small finite region of the wing, so the solution may be considered to be of the locally linearized form. More generally, then, the solution of equation (A-2) for a distribution of doublets is given by

$$
\varphi_{0}(x, y, z ; M)=\iint_{(x-\xi)>0} \rho(\xi, \eta) \varphi_{d}(x-\xi, y-\eta, z ; M) d \xi d \eta, x>0,
$$

where $\rho(x, y)$ is the doublet strength. It has been shown in reference 5 for $M=1.0$ that

$$
\rho(x, y)=\varphi_{0}\left(x, y, 0^{+}\right)
$$

From equation (A-7), after taking the partial derivative with respect to $z$, one obtains

$$
\varphi_{o_{z}}(x, y, z)=\iint_{(x-\xi)>0} \rho(\xi, \eta) \psi(x-\xi, y-\eta, z ; M) d \xi d \eta,
$$

where

$$
\psi\left(x, y, 0^{+} ; M\right)=\left.\frac{\partial \varphi_{d}}{\partial z}\right|_{z \rightarrow 0^{+}}=\left\{\begin{array}{l}
0, x \leq 0 \\
\frac{i k}{2 \pi} \frac{M^{2}}{x^{2}} \exp \left[-\frac{1}{2} i k\left(x+\frac{M^{2} y^{2}}{x}\right)\right], x>0 .
\end{array}\right.
$$

In a manner parallel to that for the linearized tangency condition, equation (A-9) may be written as

$$
w(x, y)=\iint_{S+W} \varphi_{0}\left(\xi, \eta, 0^{+}\right) \psi\left(x-\xi, y-\eta, 0^{+} ; M\right) d \xi d \eta
$$


where $w(x, y)=\varphi_{0}\left(x, y, 0^{+}\right)$

The doublet strength $\rho$ can be determined from equation $(A-9)$, or by

$$
\int_{S+W} \varphi_{0}\left(x, y, 0^{+}\right) \psi(x-\xi, y-\eta ; M) d \xi d \eta=w(x, y), \text { for }(x, y) \text { in } S
$$

and

$$
\left(\frac{\partial}{\partial x}+i k\right) \varphi_{0}\left(x, y, 0^{+}\right)=0, \text { for }(x, y) \text { in } W
$$

For a wing with an unswept trailing edge, equation $(A-12)$ becomes

$$
\iint_{S} \varphi_{0}\left(x, y, 0^{+}\right) \psi(x-\xi, y-\eta ; M) d \xi d \eta=w(x, y), \text { for }(x, y) \text { in } S
$$

Thus, the doublet potential strength $\varphi_{0}$ can be evaluated with a predescribed downwash distribution $w$ on the wing planform.

\section{DOUBLET SONIC-BOX METHOD}

The concept or scheme for obtaining the approximate solution to equation $(A-14)$ described in this section is essentially the same as that used in reference 5 . The only difference is the inclusion of the local Mach number in the integrand of the present case.

For a wing lying close to the $x y$-plane, the planform (S) is replaced by a grid of square boxes $(E)$ with their centers lying in $S$. Within each box, represented by its center, the flow properties and downwash are considered to be constant. The box having a center at $\left(x_{i}, y_{i}\right)$ is designated by $E_{i j}$. With this replacement, equation $(A-14)$ yields a system of linear algebraic equations for the $\varphi_{o_{i j}}$ 's as follows:

$$
\sum_{i^{\prime}, i^{\prime}} \varphi_{o^{\prime} i^{\prime}} \int_{E_{i} i^{\prime}} \int \psi\left(x_{i}-\xi, y_{i}-\eta ; M\right) d \xi d \eta=w\left(x_{i}, y_{i}\right)
$$

where $M$ is considered to be a parameter only.

Defining $A$ as 


$$
\begin{aligned}
A & =\int_{E_{i^{\prime} i^{\prime}}} \psi\left(x_{i}-\xi, y_{i}-\eta ; M\right) d \xi d \eta \\
& =\frac{i k}{2 \pi} M^{2} \iint_{E_{i} i^{\prime}} \frac{1}{\left(x_{i}-\xi\right)^{2}} \exp \left\{-\frac{1}{2} i k\left[\left(x_{i}-\xi\right)+\frac{M^{2}\left(y_{i}-\eta\right)^{2}}{\left(x_{i}-\xi\right)}\right]\right\} d \xi d \eta
\end{aligned}
$$

and introducing

$$
\begin{aligned}
& u=(x-\xi) / H \\
& v=M(y-\eta) / H \\
& m=\left|i-i^{\prime}\right| \\
& n=i-i^{\prime} \\
& \imath=k H
\end{aligned}
$$

into equation $(A-16)$, one obtains

$$
A=\frac{i k}{2 \pi} M \iint \frac{1}{u} \exp \left[-\frac{1}{2} i \ell\left(u+\frac{v^{2}}{u}\right)\right] d u d v
$$

where integration limits are

$$
\begin{array}{ll}
u_{1}=\frac{x-\xi}{H}-\frac{1}{2}=\left(i-i^{\prime}\right)-\frac{1}{2} & =n-\frac{1}{2} \\
u_{2}=\left(i-i^{\prime}\right)+\frac{1}{2} & =n+\frac{1}{2} \\
v_{1}=M\left[\frac{y-n}{H}-\frac{1}{2}\right]=M\left[\left(i-i^{\prime}\right)-\frac{1}{2}\right] & =M\left[m-\frac{1}{2}\right] \\
v_{2}=M\left[\left(i-i^{\prime}\right)+\frac{1}{2}\right] & =M\left[m+\frac{1}{2}\right]
\end{array}
$$

Since the integration in equation $(A-17)$ is identical to that formulated by Rodemich and Andrew (ref. 5), the integration method developed in reference 5 can be adopted directly. The only difference is the inclusion of the local Mach number in equation $(A-17)$ which reduces identically to the expression used in reference 5 when $M \rightarrow 1$ everywhere on the wing. 
In order to maintain interchangeability of the sending and the receiving points and to minimize the accumulated error of integration around the boxes instead of the wing planform, the value of $M$ in the expression $v$ is considered to be that of the arithmetic mean of receiving and the sending points, i.e.,

$$
M=\frac{1}{2}[M(x, y)+M(\xi, \eta)] .
$$

The value of $M$ outside the integration in equation ( $A-17$ ) may be similarly taken as the mean value as shown in equation $(A-18)$; but it may be adequate or even more appropriate to let $M=M(\xi, \eta)$, i.e., the Mach number of the sending points. This observation is based on inspection of equation $(A-15)$ where it is seen that the local velocity potential is modified only by the corresponding local Mach number at the center of the $i^{\prime} j$ '-th box.

All the equations required for carrying out the calculation of the transonic aerodynamic force coefficients with thickness effects have been reduced to the forms similar to those used in the original sonic-box method computer program (ref. 5). Thus it can be quite easily modified to obtain numerical results with thickness effects without using the coordinate transformation technique described in the main body of the present report. The method described herein has avoided the difficulty of the fold-over wing problem encountered previously, but the basic restrictions still apply. For example, the Mach number distribution on the wing must not be very different from that of the freestream to enable the local linearization concept to be applied; and the mean angle of attack, the camber of wing and the amplitude of oscillation must also be small so that the linearized tangency condition can be used. The mean local Mach number is cansidered to be a parameter, only, which cannot alter the wave characteristics of the disturbances in unsteady transonic flow.

\section{CONCLUDING REMARKS}

The approach described applies the local linearization method to obtain an approximation to wing thickness effects as represented by the mean local Mach number in the unsteady transonic flow analysis. The basic solution of the governing small-perturbation velocitypotential equation is modified by the mean local Mach number to account for thickness effects.

The previous method for accomplishing this required a coordinate transformation which, far certain wings, resulted in a non-one-to-one wing planform transformation. It is shown that this new method for introducing the local Mach number into the sonic-box computer program does not have this limitation. 
The equations to be used in the calculation of the aerodynamic force coefficients with thickness effects have been reduced to those forms used in the original sonic-box computer program; thus, only a minor modification to the original computer program is required to obtain numerical results. 


\section{REFERENCES}

1. Ferrari, C.; and Tricomi, F. G.: Transonic Aerodynamics. Academic Press, New York, 1968.

2. Landahl, M. T.: Unsteady Transonic Flow. Pergamon Press, New York, 1961.

3. Davies, D. E.: Three Dimensional Sonic Theory. AGARD Manual on Aeroelastic ity, Part II - Aerodynamic Aspect, Chapter 4.

4. Kacprzynski, J.. J.; Ashley, H.; and Sankaranarayanan, R.: On the Calculation of Unsteady Nonlinear Three-Dimensional Supersonic Flow Past Wings. J. of Basic Eng. , Transactions of ASME, vol. 90, no. 4, Dec. 1968, pp. 581-595.

5. Rodemich, E. R.; and Andrew, L. V.: Unsteady Aerodynamics for Advanced Configurations, Part II - A Transonic Box Method for Planar Lifting Surfaces. FDL-TDR-64152, Part II, May 1965, Air Force Flight Dynamics Lab., Wright-Patterson Air Force Base, Ohio.

6. Ruo, S. Y.: Calculation of Unsteady Transonic Aerodynamics for Oscillating Wings with Thickness (Computer Program), NASA CR-132477, Sept. 1974.

7. Bisplinghoff, R. L.; Ashley, H.; and Halfman, R.L.: Aeroelasticity. AddisonWesley, 1955, p. 193.

8. Lin, C. C.; Reissner, E.; and Tsien, H. S.: On the Two-Dimensional Non-Steady Motion of a Slender Body in a Compressible Fluid. J. Math. Phys., vol. XXVII, 1948, pp. 220-231.

9. Miles, J. W.: The Potential Theory of Unsteady Supersonic Flow. Cambridge University Press, 1959.

10. Lamb, H.: Hydrodynamics. Cambridge University Press, First American Ed., 1945, p. 7.

11. Spreiter, J.R.; and Alksne, A.: Theoretical Prediction of Pressure Distributions on Nonlifting Airfoils at High Subsonic Speeds. NACA Rept. 1217, 1955.

12. Oswatitsch, K.; and Berndt, S. B.: Aerodynamic Similarity at Axisymmetric Transonic Flow Around Slender Bodies. KTH Aero TN 15, 1950, Royal Inst. Tech., Stockholm, Sweden.

13. Busemann, A.: Application of Transonic Similarity. NACA TN 2687, 1952. 
14. Spreiter, J. R.: On the Application of Transonic Similarity Rules. NACA Rept. $1153,1953$.

15. Maeder, P.F.; and Thommen, H. U.: Some Results of Linearized Transonic Flow About Slender Airfoils and Bodies of Revolution. J. Aero. Sci., vol. 23, no. 2, Feb. 1956, pp. 187-188.

16. Berndt, S. B.: Similarity Laws for Transonic Flow Around Wings of Finite Aspect Ratio. KTH Aero TN 14, 1950, Royal Inst. Tech., Stockholm, Sweden.

17. Oswatitsch, K.: Die Geschwindigkeitsverteilung bei lokalen Überschallgebieten an flachen Profilen. Zeitschrift für Angewandte Mathematik und Mechanik. bd. 30, nr. 1/2, Jan./Feb. 1950, s. 17-24.

18. Oswatitsch, K.: Die Gerschwindigkeitsverteilung an Symmetrischen Profilen beim Auftreten lokaler Überschallgebiete. Acta Physica Austriaca, bd. 4, nr. 2-3, Dec. 1950, s. 228-271.

19. Oswatitsch, K.; and Keune, F.: Flow Around Bodies of Revolution at Mach Number One. Proceedings of the Conference on High-Speed Aeronautics, Polytechnic Institute of Brooklyn, Jan. 20-22, 1955, pp. 113-131.

20. Hosokawa, 1.: A Refinement of the Linearized Transonic Flow Theory. J. Phy. Soc. Japan, vol. 15, no. 11, 1960, pp. 149-157.

21. Cole, J. D.; and Royce, W. W.: An Approximate Theory for the Pressure Distribution and Wave Drag of Bodies of Revolution at Mach Number One. Proceedings 6th Midwestern Conference on Fluid Mechanics, 1960, pp. 254-276.

22. Miles, J. W.: Linearization of the Equations of Non-Steady Flow in a Compressible Fluid. J. of Mathematics and Physics, vol. 33, no. 2, July 1954, p. 135.

23. Mangler, K. W.: A Method of Calculating the Short Period Longitudinal Stability Derivatives of a Wing in Linearized Unsteady Compressible Flow. British A.R.C. $R$ \& M 2924, 1952.

24. Miles, J. W.: On the Low Aspect Ratio Rectangular Wing in Supersonic Flow. Aeronautical Quarterly, 4, pt. 3, 1953, pp. 231-244.

25. Runyan, H. L.; and Woolston, D. S.: Method for Calculating the Aerodynamic Loading on an Oscillating Finite Wing in Subsonic and Sonic Flow. NACA Report 1322., 1957.

26. Albano, E.; and Andrew, L. V.: Unsteady Aerodynamics for Advanced Configurations, Part III - Elliptic-Conical Wing in Linearized Unsteady Transonic. Flow. FDL-TDR64-152, Part III, May 1965, Air Force Flight Dynamics Lab., Wright-Patterson Air Force Base, Ohio. 
27. Stenton, T. E.; and Andrew, L. V.: Transonic Unsteady Aerodynamics for Planar Wings with Trailing Edge Control Surfaces. AFFDL-TR-67-180, Aug. 1968, Air Force Flight Dynamics Lab., Wright-Patterson Air Force Base, Ohio.

28: Teipel, 1.: Die Instationären Luftkräfte bei der Maczahl 1. Z. Flugwiss, 12, heft 1, 1964.

29. Liu, D. D.; Platzer, M. F.; and Ruo, S. Y.: On the Calculation of Static and Dynamic Stability Derivatives for Bodies of Revolution at Subsonic and Transonic Speeds. AlAA Paper No. 70-190, 1970.

30. Ruo, S. Y.; and Liu, D. D.: Calculation of Stability Derivatives for Slowly Oscillating Bodies of Revolution at Mach 1.0. LMSC/HREC D162375, Feb. 1971, Lockheed Missiles and Space Co., Huntsville, Alabama.

31. Spreiter, John R.; and Alksne, Alberta Y.: The Airfoil Theory Based on Approximate Solution of the Transonic Flow Equation. NACA Report 1359, 1958.

32. Rubbert, P. E.; and Landahl, M. T.: Solution of the Transonic Airfoil Problem through Parametric Differentiation. AlAA J., vol. 5, no. 3, Mar. 1967, pp. 470-479.

33. Landahl, M. T.: Linearized Theory for Unsteady Transonic Flow. MIT-FDRL-Rep. No. 63-2, March 1963.

34. Ashley, H.: Machine Computation of Aerodynamic Loads in Linear and Nonlinear Situations. Proc. Ninth Indian Congress of Applied Mechanics, Kanpur, 1963 (also MIT-FDLR-Rep. 66-5).

35. Sankaranarayanan, R.; and Vijayavittal, C. S.: Effect of Thickness on Wings in Supersonic Flow. J. Aero. Soc. of India, vol. 22, no. 2, May 1970, pp. 108-117.

36. Kacprzynski, J. J.: On the Calculation of Unsteady Nonlinear Three-Dimensional Supersonic Flow Past Wings. AFOSR-68-0517, SUDAAR No. 339, 1968.

37. Andrew, L. V.; and Stenton, T. E.: Unsteady Aerodynamics for Advanced Configurations, Part VII - Velocity Potentials in Non-Uniform Transonic Flow Over a Thin Wing. FDL-TDR-64-152, Part VII, August 1968, Air Force Flight Dynamics Lab., Wright-Patterson Air Force Base, Ohio.

38. Garrick, I. E.; and Kaplan, C.: On the Flow of a Compressible Fluid by the Hodograph Method I - Unification and Extension of Present-Day Results. NACA Report No. 789, 1944.

39. Garrick, 1. E.; and Kaplan, C.: On the Flow of a Compressible Fluid by the Hodograph Method II - Fundamental Set of Particular Flow Solutions of the Chaplyg in Differential Equation. NACA Report No. 790, 1944. 
40. Guderley, G.: On Transonic Airfoil Theory. J. Aero. Sci., vol. 23, no. 10, Oct. 1956, pp. $961-969$.

41. Alksne, A.Y.; and Spreiter, J.R.: Theoretical Pressure Distributions on Wings of Finite Span of Zero Incidence for Mach Numbers Near 1. NASA Report R-88, 1960.

42. Orlik-Rückemann, K.; and Olsson, C. O.: A Method for the Determination of the Damping-in-Pitch of Semi-Span Models in High-Speed Wind-Tunnels and Some Results for a Triangular Wing. Swedish F.F.A. Report 62, 1956.

43. Statler, I. C.: Dynamic Wind-Tunnel Tests of a Delta-Wing Model at Transonic Speeds. AFFDL-TR-69-97, January 1970, Air Force Flight Dynamics Lab., WrightPatterson Air Force Base, Ohio.

44. Olsen, J. J.: Demonstration of a Transonic Box Method for Unsteady Aerodynamics of Planar Wings. AFFDL-TR-66-121, Oct. 1966, Air Force Flight Dynamics Lab., Wright-Patterson Air Force Base, Ohio.

45. Hosokawa, I.: A Simplified Analysis for Transonic Flow. IUTAM Symposium Transsonicum, K. Oswatitsch, ed., Springer-Verlag (Berlin), 1964, pp. 184-199.

46. Kimble, K. R.; Liu, D. D.; Ruo, S. Y.; and Wu, J. M.: Unsteady Transonic Flow Analysis for Low Aspect Ratio, Pointed Wing. AIAA J., vol. 12, no. 4, Apr. 1974, pp. 516-522. 


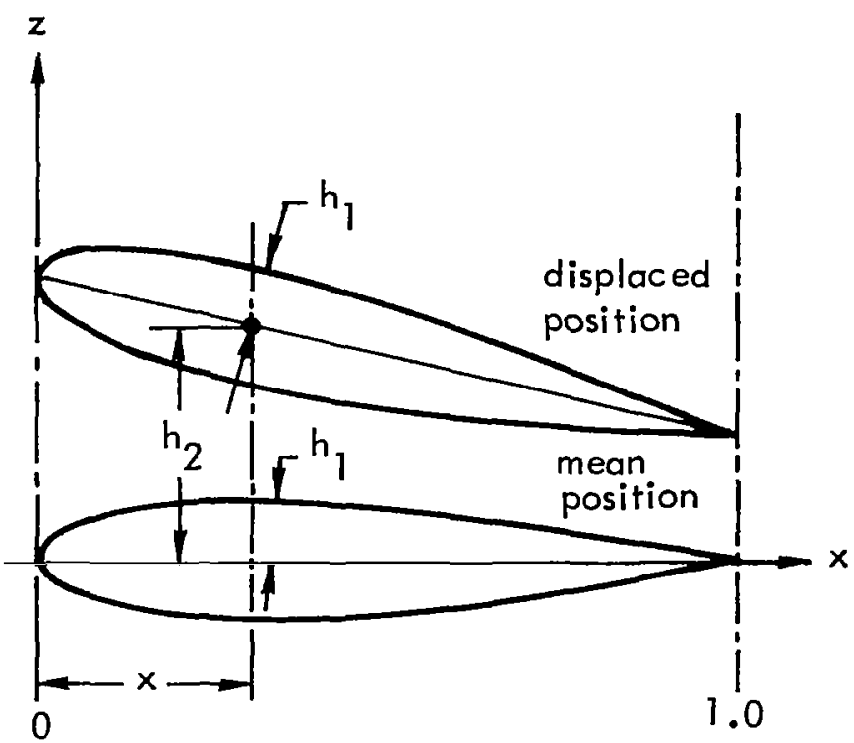

Figure 1.- Wing displacement.

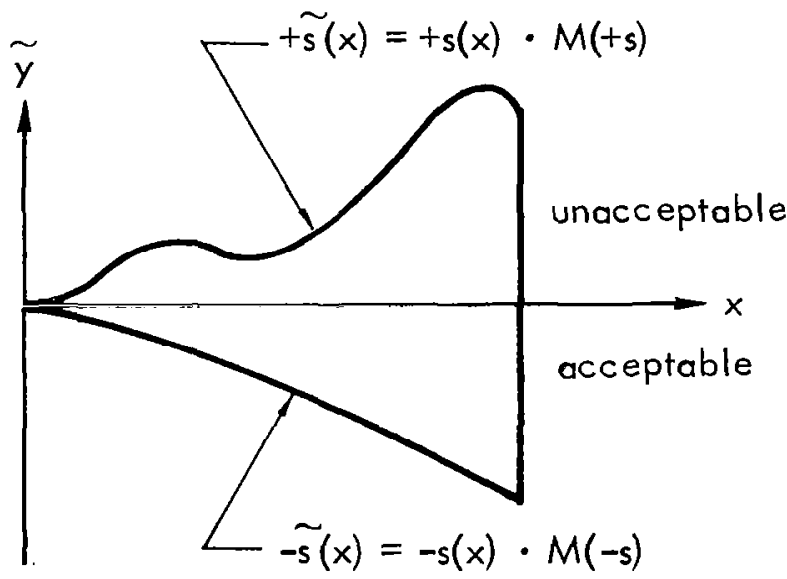

Figure 2.- Transformation of leading edge. 


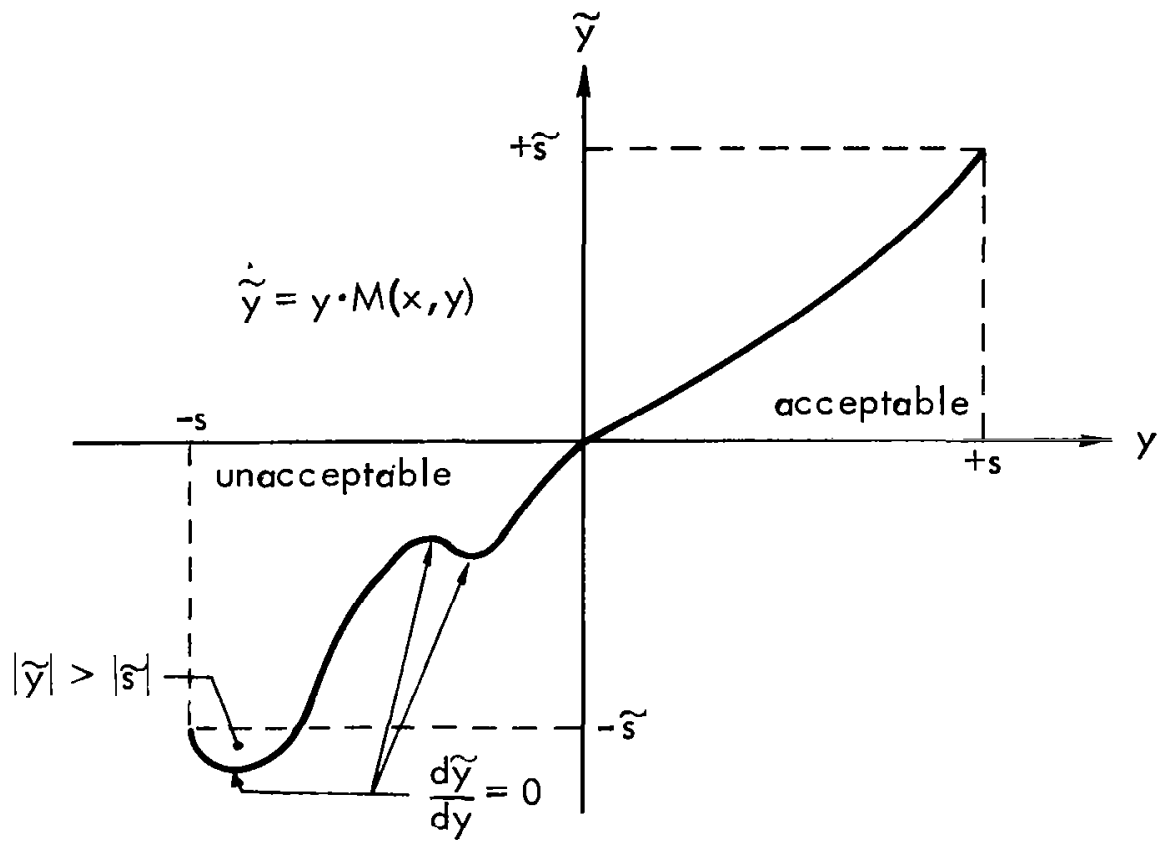

Figure 3.- Transformation of spanwise coordinate.

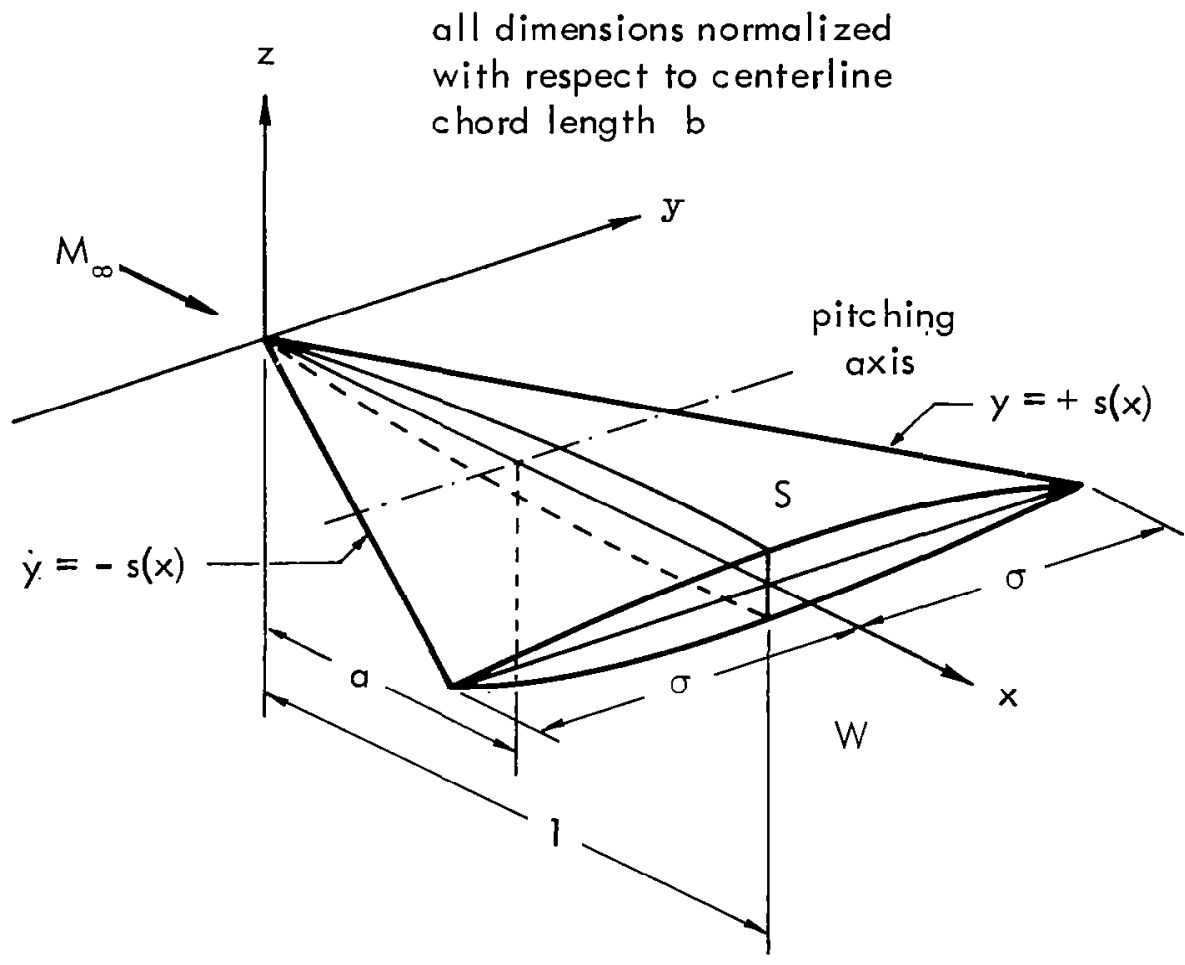

Figure 4.- Physical coordinates and sample wing geometry. 

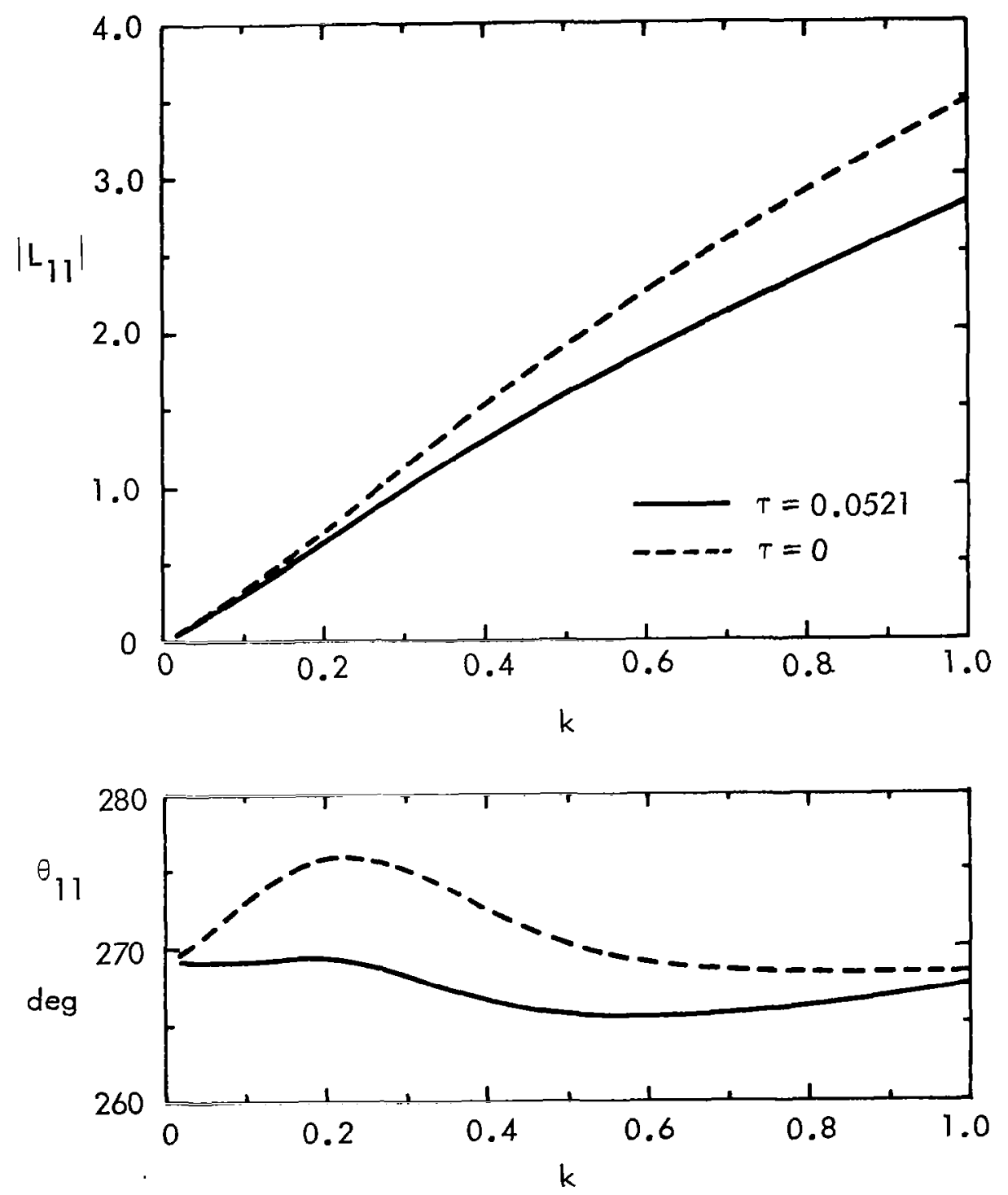

(a). Lift and phase angle due to plunge.

Figure 5.- Generalized aerodynamic force coefficients for a rectangular wing of aspect ratio 2.0 with $a=0$ and 25 boxes chordwise. 

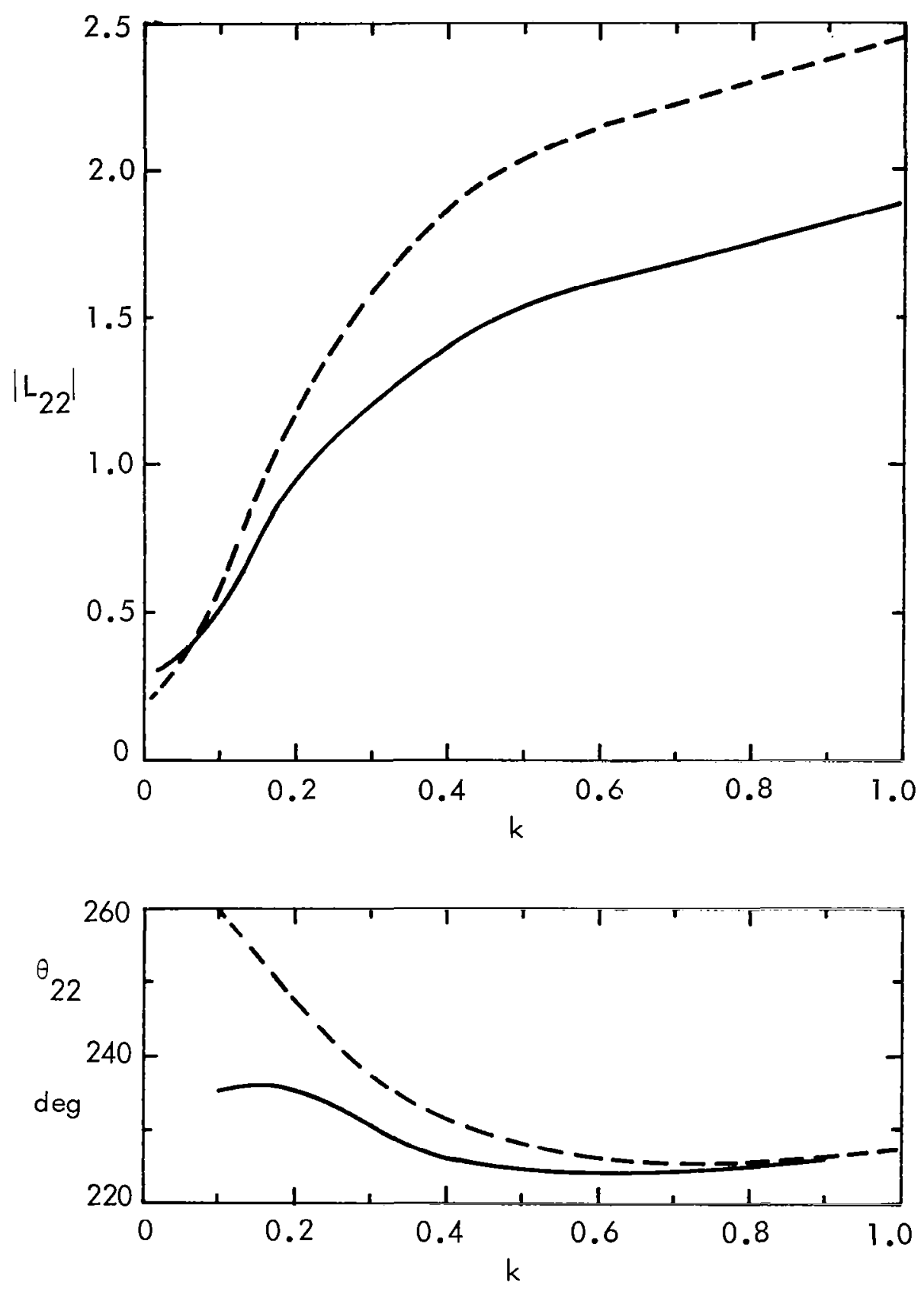

(b). Pitching moment and phase angle due to pitch.

Figure 5.- Concluded 

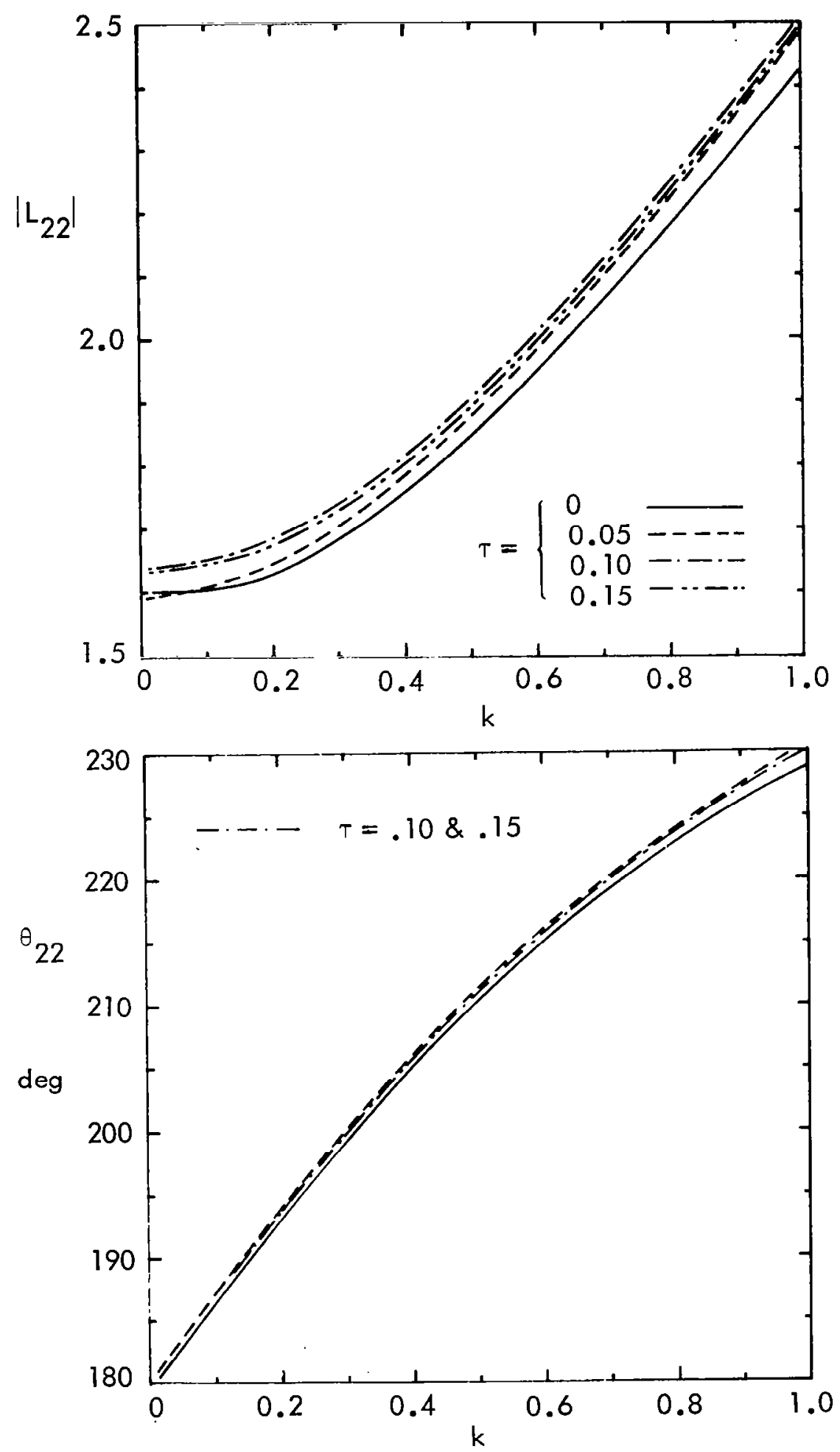

Figure 6.- Generalized aerodynamic force coefficients for pitching moment due to pitch of a delta wing of aspect ratio 1.5 with $a=0$ and 40 boxes chordwise. 


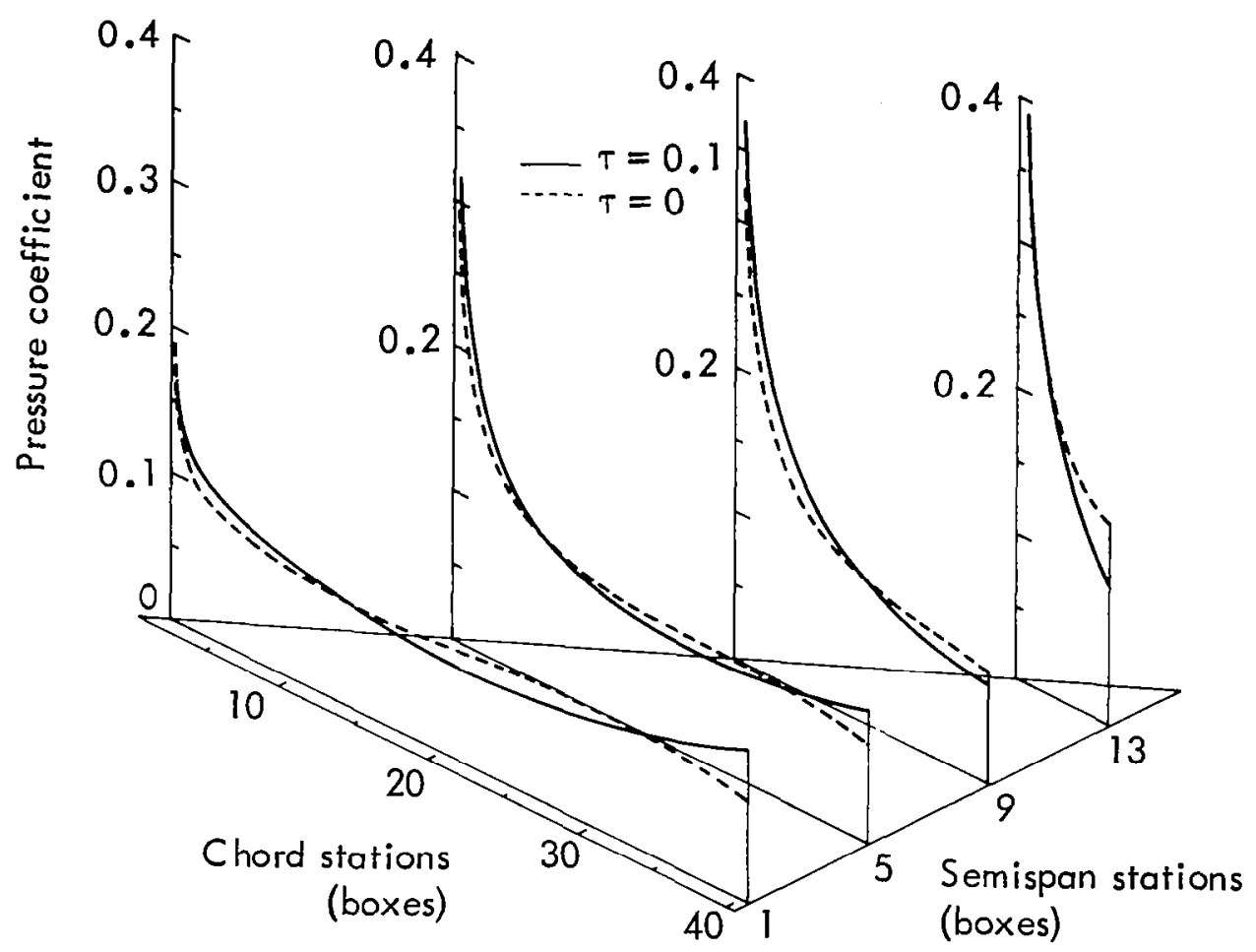

(a). Plunge mode.

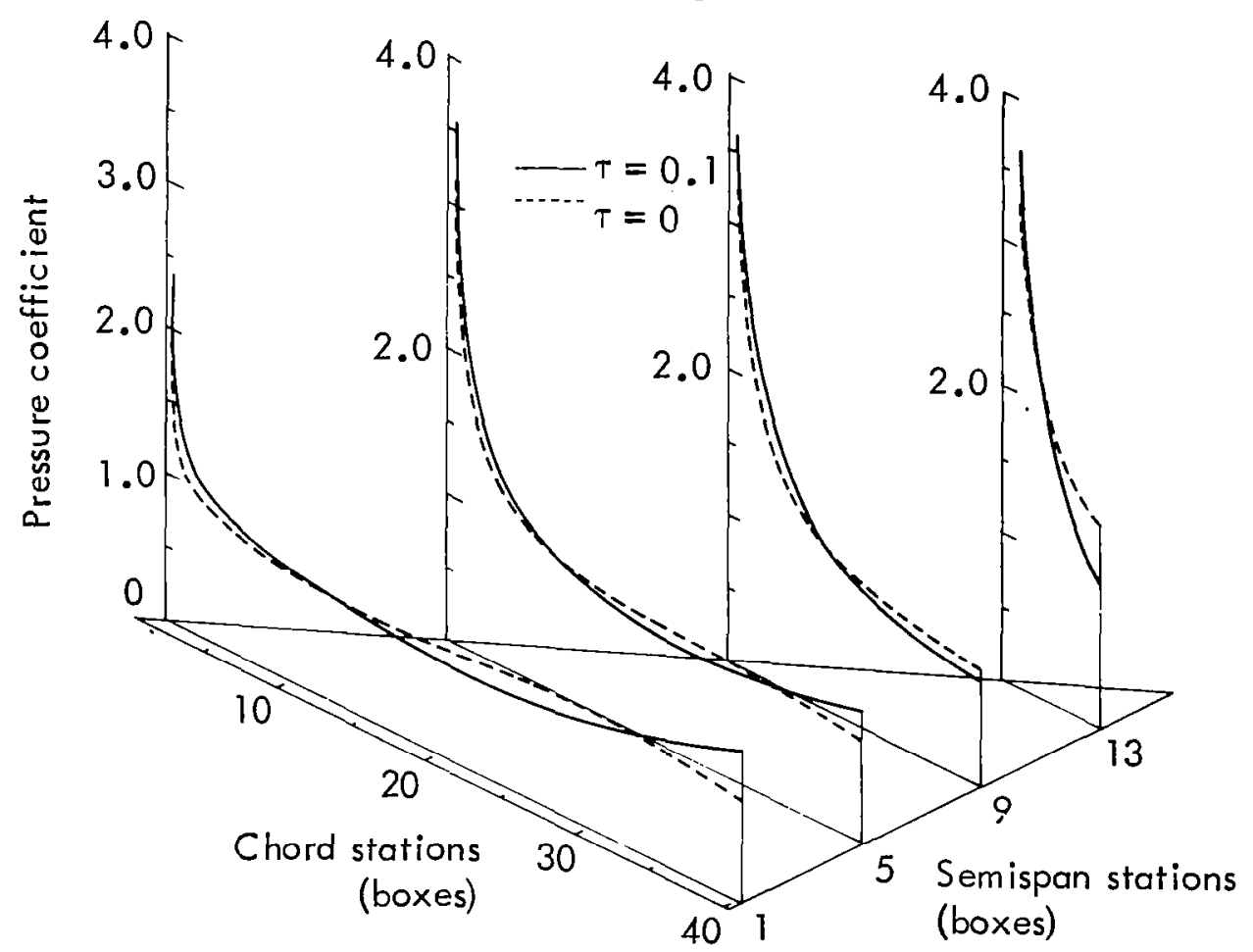

(b). Pitch mode with $a=0$.

Figure 7. - Unsteady pressure for a delta wing of aspect ratio 1.5 with $k=0.1$. 


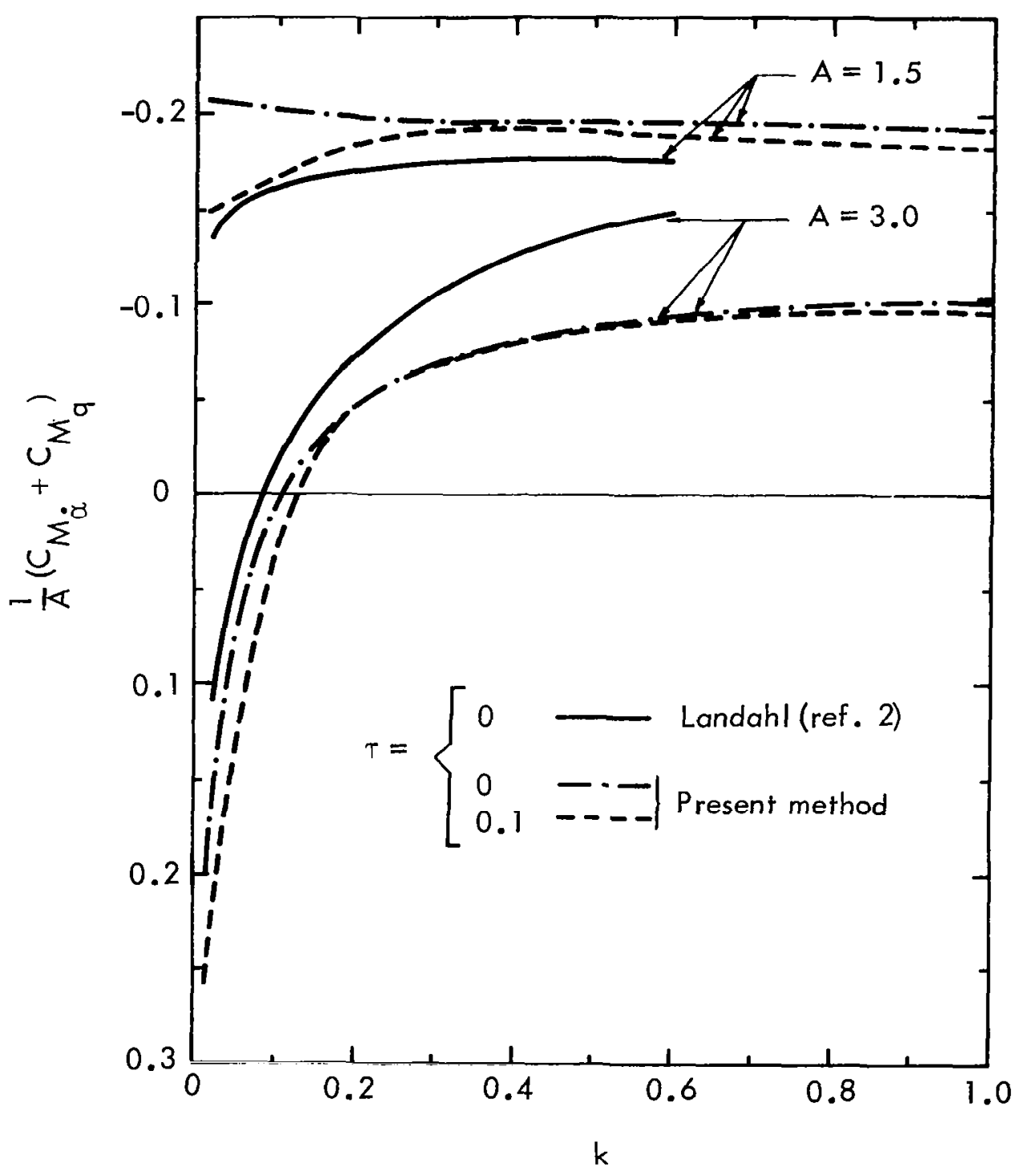

Figure 8. - Comparison of present method for delta wing pitch-damping derivatives with results by earlier thin-wing methods. $a=0.6$. 


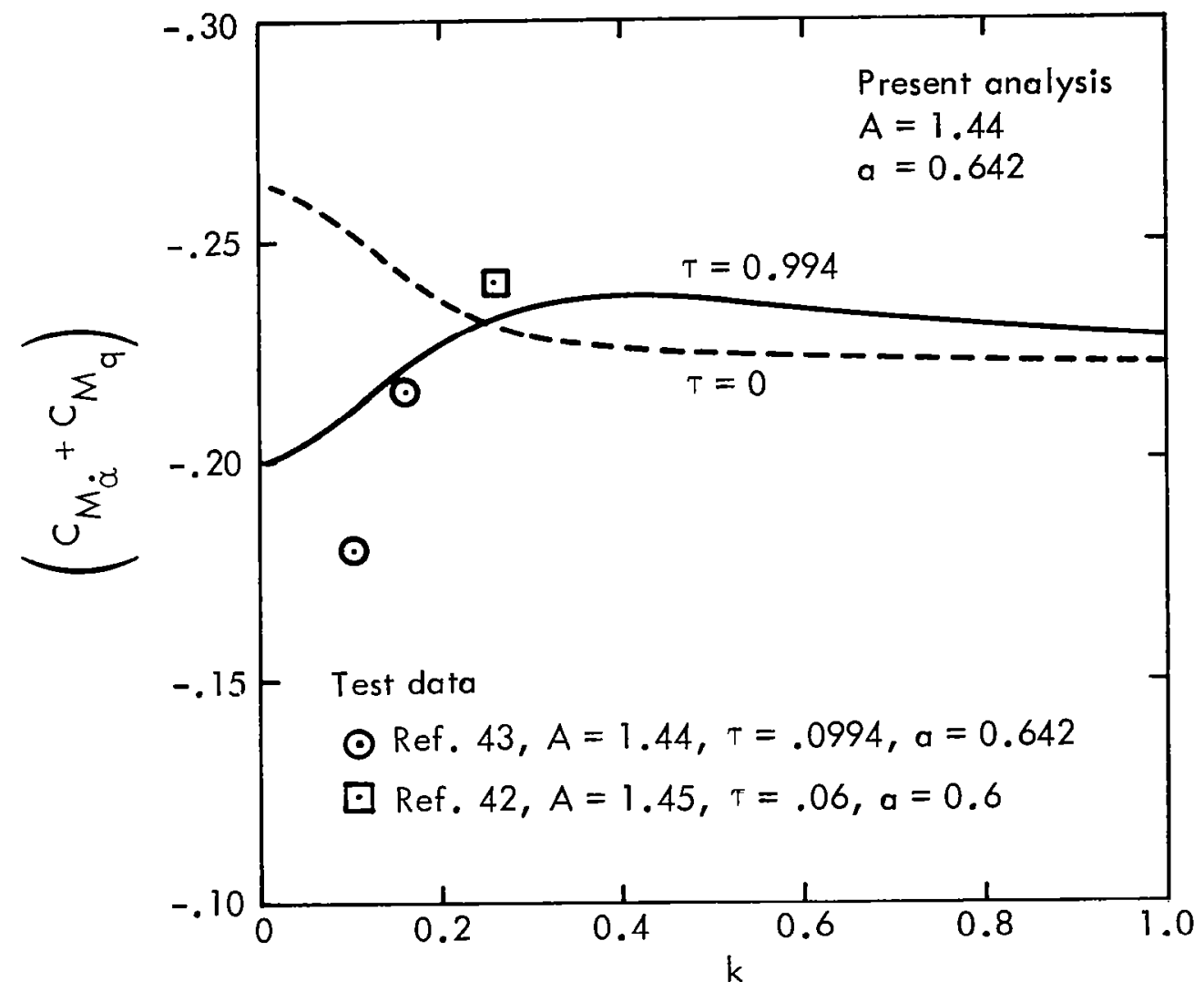

Figure 9.- Comparison of present analysis for pitching-damping derivatives with delta-wing test data at various wing thicknesses. 


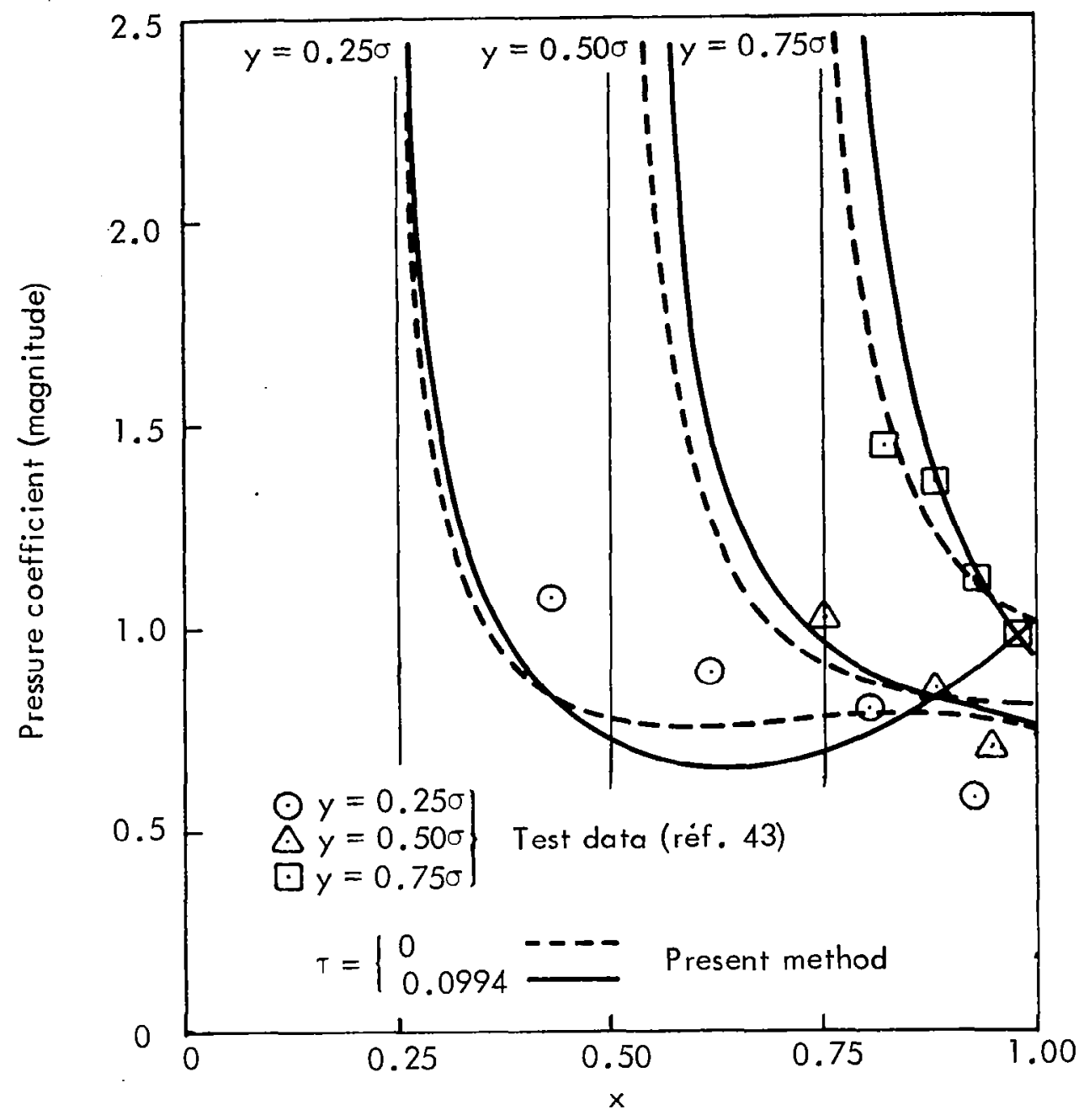

Figure 10.- Comparison of chordwise pressure variations with test data at three spanwise stations on a delta wing of aspect ratio 1.442 with $k=0.108$. 


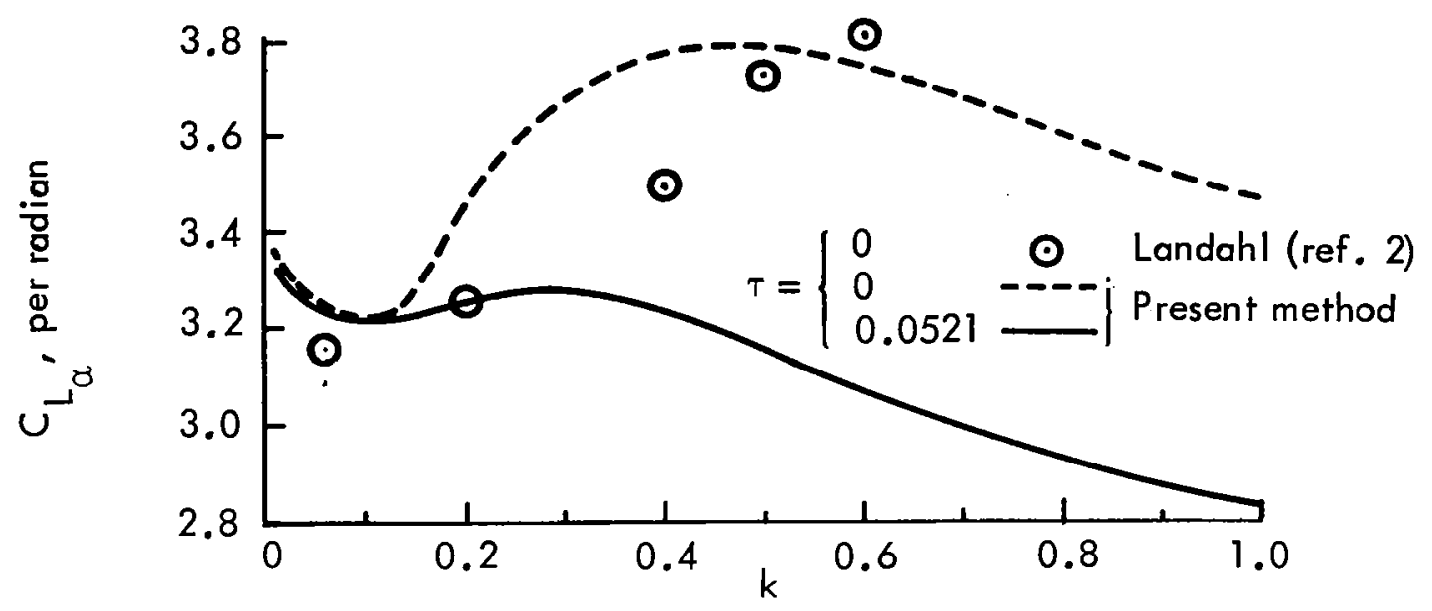

(a). Rectangular wing of aspect ratio 2.0
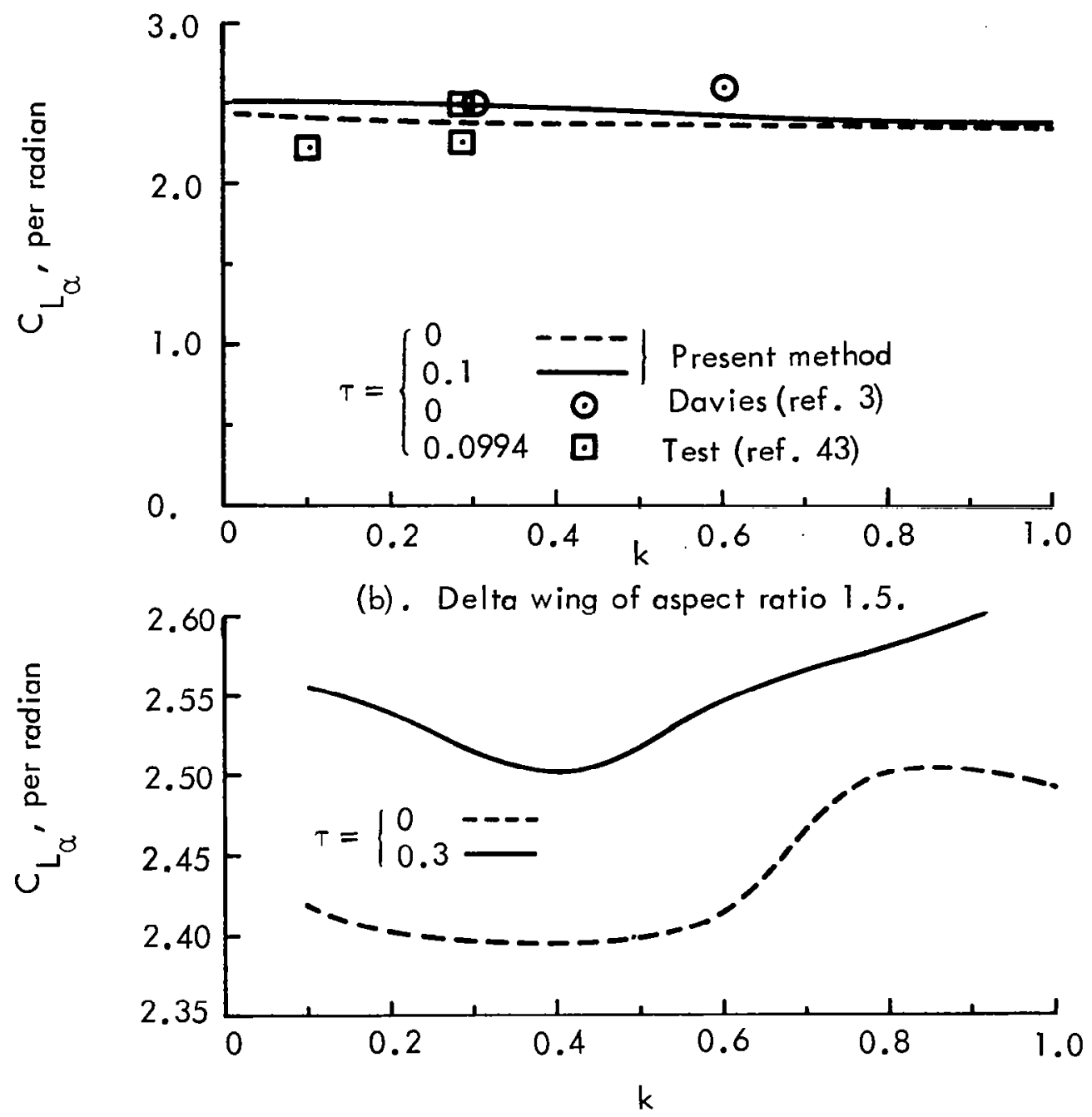

(c). Parabolic wing of aspect ratio 1.5.

Figure 11.- Lift-curve slope variation with reduced frequency and thickness for three wing planforms. 

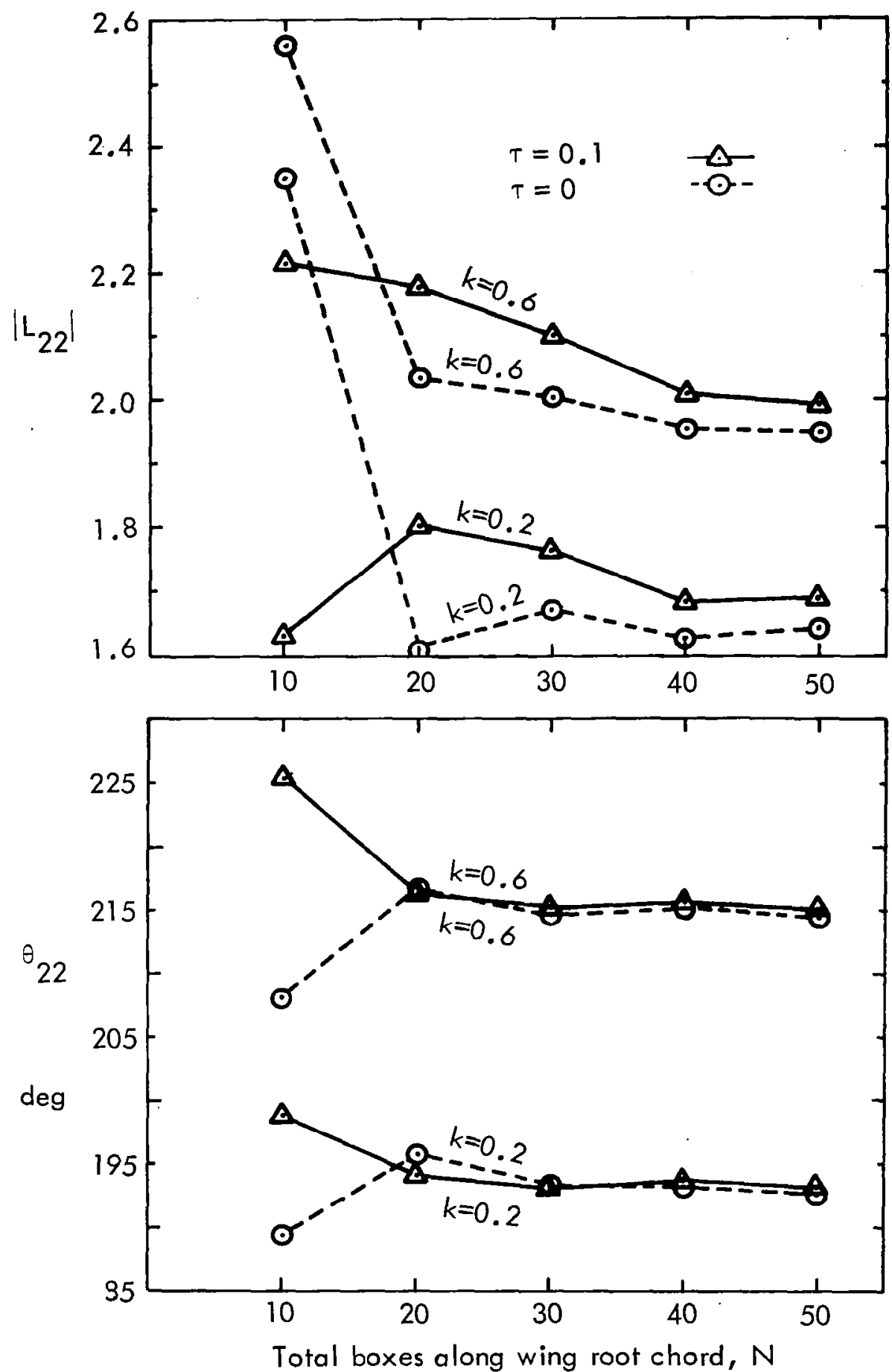

Figure 12.- Convergence of pitching moment due to pitch for delta wing of aspect ratio 1.5 with $a=0$. 